QUARTERLY OF APPLIED MATHEMATICS

VOLUME LXVIII, NUMBER 3

SEPTEMBER 2010, PAGES 563-590

S 0033-569X(2010)01189-1

Article electronically published on June 4, 2010

\title{
HILBERT PROBLEM FOR A MULTIPLY CONNECTED CIRCULAR DOMAIN AND THE ANALYSIS OF THE HALL EFFECT IN A PLATE
}

\author{
BY \\ Y. A. ANTIPOV (Department of Mathematics, Louisiana State University, Baton Rouge, Louisiana \\ $70803)$ \\ AND \\ V. V. SILVESTROV (Department of Mathematics, Gubkin Russian State University of Oil and Gas, \\ Moscow 119991, Russia)
}

\begin{abstract}
In this paper we analyze the Hilbert boundary-value problem of the theory of analytic functions for an $(N+1)$-connected circular domain. An exact series-form solution has already been derived for the case of continuous coefficients. Motivated by the study of the Hall effect in a multiply connected plate we extend these results by examining the case of discontinuous coefficients. The Hilbert problem maps into the Riemann-Hilbert problem for symmetric piece-wise meromorphic functions invariant with respect to a symmetric Schottky group. The solution to this problem is derived in terms of two analogues of the Cauchy kernel, quasiautomorphic and quasimultiplicative kernels. The former kernel is known for any symmetric Schottky group. We prove the existence theorem for the second (quasimultiplicative) kernel for any Schottky group (its series representation is known for the first class groups only). We also show that the use of an automorphic kernel requires the solution to the associated real analogue of the Jacobi inversion problem, which can be bypassed if we employ the quasiautomorphic and quasimultiplicative kernels. We apply this theory to a model steady-state problem on the motion of charged electrons in a plate with $N+1$ circular holes with electrodes and dielectrics on the walls when the conductor is placed at a right angle to the applied magnetic field.
\end{abstract}

1. Introduction. Let $D(\ni \infty)$ be an $(N+1)$-connected domain, a complex $z$-plane with $N+1$ holes bounded by Lyapunov contours $L_{\nu}(\nu=0,1, \ldots, N)$, and let $a(t), b(t)$, and $c(t)$ be some prescribed real functions Hölder-continuous on the contour $L=\cup_{\nu=0}^{N} L_{\nu}$.

Received December 4, 2008.

2000 Mathematics Subject Classification. Primary 30E25, 32N15; Secondary 74F15.

Key words and phrases. Riemann-Hilbert problem, automorphic functions, Schottky group, Hall effect. The first author was supported in part by NSF Grant DMS0707724.

The second author was supported in part by Russian Foundation for Basic Research Grant 07-01-00038. E-mail address: antipov@math.1su.edu 
The second fundamental boundary-value problem of the theory of analytic functions, the Hilbert problem, requires the finding of all functions $\phi(z)$ that are single-valued and analytic in $D$, Hölder-continuous up to the boundary $L=\cup_{\nu=0}^{N} L_{\nu}$, and that satisfy the boundary condition

$$
\operatorname{Re}[\overline{f(t)} \phi(t)]=c(t), \quad t \in L
$$

where $f(t)=a(t)+i b(t)$.

If at least one of the contours $L_{\nu}$ is not a circle, and $N \geq 1$, then the Hilbert problem cannot be solved exactly. In this case, by the method of the regularizing Schwarz factor [22], [15, it can be reduced to a system of singular integral equations. Alternatively, the solution can be expressed through a basis of $N+1$ complex harmonic measures [26]. This method, in addition to the Schwarz factor, uses the theory of the Riemann-Hilbert problem on a Riemann surface and requires the solution to a real analogue of the classical Jacobi inversion problem.

In the case when all the contours $L_{\nu}$ are circles, and the functions $a(t), b(t)$, and $c(t)$ are Hölder-continuous on $L$, the Hilbert problem admits an exact solution in a series form [1, 2], 20]. One of the ways to solve the problem in this case is to convert the original Hilbert problem into a Riemann-Hilbert problem for symmetric piece-wise meromorphic functions invariant with respect to a Schottky group of symmetric Möbius transformations [20]. This idea was used in the study of steady-state flow around $N+1$ cylinders with porous walls [4. Its solution requires the analysis of a Riemann-Hilbert problem with continuous coefficients. Recently [5], the method was extended to free boundary problems on supercavitating flow in multiply connected domains. The key step in the solution procedure is the determination of a conformal mapping in terms of the solutions to two Hilbert problems for a multiply connected circular domain. The first problem has continuous coefficients, whilst the second one is a homogeneous problem with the coefficient

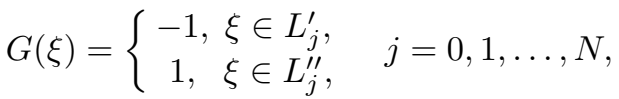

where $L_{j}=L_{j}^{\prime} \cup L_{j}^{\prime \prime}$ and $L_{j}$ are circles.

In the present paper, motivated by an electromagnetic problem for a Hall semiconductor with $N+1$ circular holes, we analyze the general case of the Hilbert problem (1.1) with discontinuous functions $a(t), b(t)$, and $c(t)$. The actual physical problem is homogeneous, and the coefficients $a(t)$ and $b(t)$ are discontinuous functions. The discontinuity is caused by the presence of electrodes and dielectrics on the walls of the holes. Due to the generalized Ohm's law describing the Hall effect, the boundary conditions on the electrodes and the dielectrics, $E_{\tau}=0$ and $J_{n}=0$, respectively, and the Maxwell equations give rise to a particular case of the Hilbert problem with piece-wise continuous coefficients $a(t)$ and $b(t)$. Here $E_{\tau}$ is the tangent component of the electric field intensity, and $J_{n}$ is the normal component of the current intensity.

Various authors [25], 16], 17], 23], 24], 12] investigated the electrical characteristics of Hall plates. These papers adopt the method of conformal maps devised by Wick 25] for simply connected Hall plates. The method of conformal maps was further developed and numerically implemented in 21, 11] for simply connected polygonal domains. Some particular cases of a doubly connected Hall plate in the form of an annulus with a pair 
of symmetric electrodes were considered in [16, 12, where exact solutions were derived in terms of elliptic functions. To the knowledge of the authors, an analytical solution to the general case of the electromagnetic problem for a circular multiply connected Hall plate with any finite number of electrodes and dielectrics on the walls is not available in the literature.

One of the steps of the solution to the Hilbert problem with discontinuous coefficients is the factorization problem. Its solution was derived $[6$ for an $(N+1)$-connected circular domain in terms of an automorphic analogue of the Cauchy kernel. The kernel was expressed through the Schottky-Klein prime function of the associated Schottky double. This procedure requires eliminating singularities at extra poles of the kernel by solving a real analogue of the Jacobi inversion problem and normalizing the basis of abelian integrals.

In this paper we aim to derive an exact solution to the general case of the Hilbert problem (1.1) with discontinuous coefficients for an $(N+1)$-connected circular domain. First, we shall reduce the Hilbert problem (1.1) to the first fundamental problem of analytic functions, the Riemann-Hilbert problem for symmetric piece-wise meromorphic functions invariant with respect to a Schottky group. Next, we shall introduce a multiplicative canonical function and derive its representation in terms of a quasiautomorphic analogue of the Cauchy kernel (Theorem 3.5). It turns out that the use of a quasimultiplicative analogue of the Cauchy kernel for the solution of both the homogeneous and inhomogeneous problems allows us to bypass the Jacobi inversion problem. Such a kernel was derived in [20] for the first class groups (Burnside's classification [7]). Here (Theorem 4.1), by using the Riemann-Roch theorem for multiplicative functions [19, we shall prove the existence of such a kernel. Then we shall derive the general solution to the homogeneous and inhomogeneous cases of the Hilbert problem and analyze its solvability (Theorems 5.2 and 5.3). We shall also specify the solution for the first class groups. In addition, we shall solve the Hilbert problem (1.1) with discontinuous coefficients in terms of an automorphic canonical function and the solution to the associated real analogue of the Jacobi problem. Motivated by applications in electromagnetics we shall present the solution in the special case for piece-wise constant coefficients. Finally, we shall give an exact solution to a circular $(N+1)$-connected plate with electrodes and dielectrics on the walls when the applied electric and transverse magnetic fields cause the Hall effect. The solution will be presented in a series form for the first class Schottky groups.

2. Riemann-Hilbert problem with discontinuous coefficients for piece-wise automorphic symmetric functions. Let $D$ be an $(N+1)$-connected domain which is a complex $z$-plane with $N+1$ holes bounded by circles $L_{\nu}=\left\{z \in \mathbb{C}:\left|z-\delta_{\nu}\right|=\rho_{\nu}\right\}$, $\nu=0,1, \ldots, N$ (Fig. 1). The positive direction on the circle $L_{\nu}$ is chosen such that the exterior of $L_{\nu}$ is on the left. Define $L=\cup_{\nu=0}^{N} L_{\nu}$ and consider the following Hilbert problem:

Problem 2.1. Let

$$
a(t)=a_{\nu}(t) \quad \text { and } \quad b(t)=b_{\nu}(t) \quad\left(t \in L_{\nu}, \nu=0,1, \ldots, N\right)
$$




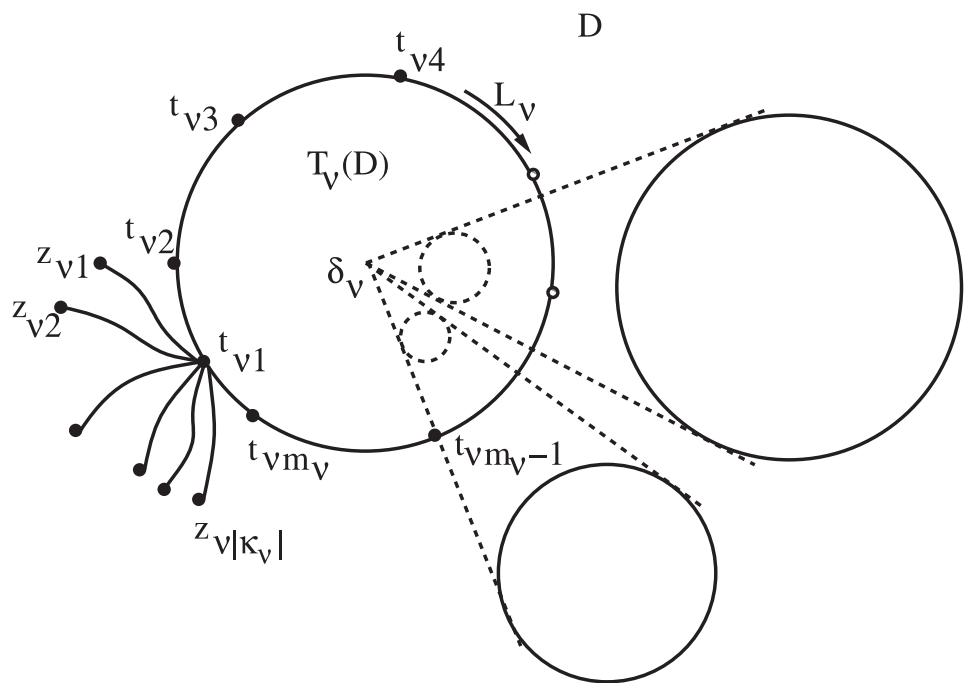

FIG. 1. The geometry of the problem.

be real functions satisfying the Hölder condition either everywhere on $L_{\nu}$, or everywhere on $L_{\nu}$ except at points $t_{\nu 1}, t_{\nu 2}, \ldots, t_{\nu m_{\nu}}, \nu=0,1, \ldots, N$, where at least one of the functions (2.1) has a jump discontinuity. Assume that $a_{\nu}^{2}(t)+b_{\nu}^{2}(t) \neq 0$ everywhere on all the contours $L_{\nu}$. Let $c(t)=c_{\nu}(t)\left(t \in L_{\nu}\right)$ be a real Hölder-continuous function on $L_{\nu}, \nu=0,1, \ldots, N$.

Find all functions $\phi(z)=u(z)+i v(z)$, holomorphic in $D$, Hölder-continuous everywhere in $D \cup L$ except at the points $t_{\nu j}\left(j=1,2, \ldots, m_{\nu}, \nu=0,1, \ldots, N\right)$, where they may have integrable singularities, are bounded at infinity and satisfy the boundary condition

$$
a(t) u(t)+b(t) v(t)=c(t), \quad t \in L \backslash \Theta,
$$

where $\Theta=\cup_{\nu=0}^{N} \cup_{j=1}^{m_{\nu}} t_{\nu j}$.

To solve this problem, we transform it into a Riemann-Hilbert problem for piecewise automorphic functions. For this, we consider the symmetry group, $\mathfrak{G}$, of the line $L=L_{0} \cup L_{1} \cup \ldots \cup L_{N}$ generated by the linear transformations $\sigma_{\nu}(z)=T_{\nu} T(z), \nu=$ $1,2, \ldots, N$, where

$$
T=T_{0}, \quad T_{\nu}(z)=\delta_{\nu}+\frac{\rho_{\nu}^{2}}{\bar{z}-\bar{\delta}_{\nu}}, \quad \nu=0,1, \ldots, N,
$$

and $T_{\nu}$ is the symmetry transformation with respect to the circle $L_{\nu}$. Denote the fundamental region of the group $\mathfrak{G}$ by $\mathfrak{F}=D \cup T(D) \cup L$. The group $\mathfrak{G}$ is a symmetry Schottky group [14. The elements of the group are the identical map $\sigma_{0}(z)=z$ and all possible compositions of the generators $\sigma_{\nu}=T_{\nu} T$ and the inverse maps $\sigma_{\nu}^{-1}=T T_{\nu}$ $(\nu=1,2, \ldots, N)$ :

$$
\begin{gathered}
\sigma \in \mathfrak{G} \Leftrightarrow \sigma=T_{k_{2 \mu}} T_{k_{2 \mu-1}} \ldots T_{k_{2}} T_{k_{1}}, \quad \mu=1,2, \ldots \\
k_{1}, k_{2}, \ldots, k_{2 \mu}=0,1, \ldots, N, \quad k_{2} \neq k_{1}, k_{3} \neq k_{2}, \ldots, k_{2 \mu} \neq k_{2 \mu-1} .
\end{gathered}
$$


The region $\mathfrak{D}=\cup_{\sigma \in \mathfrak{G}} \sigma(\mathfrak{F})$ is invariant with respect to the group $\mathfrak{G}: \sigma(\mathfrak{D})=\mathfrak{D}$ for all $\sigma \in \mathfrak{G}$. This region is symmetric with respect to all the circles $L_{\nu}(\nu=0,1, \ldots, N)$, and $\mathfrak{D}=\overline{\mathbb{C}} \backslash \Lambda$, where $\overline{\mathbb{C}}=\mathbb{C} \cup\{\infty\}$ is the extended $z$-plane, and $\Lambda$ is the set of the limit points of the group $\mathfrak{G}$ (it consists of two points if $N=1$ and it is infinite if $N \geq 2$ ). Notice that all elements of the group $\mathfrak{G}$ can be represented in the form

$$
\sigma(z)=\frac{a_{\sigma} z+b_{\sigma}}{c_{\sigma} z+d_{\sigma}}, \quad a_{\sigma} d_{\sigma}-b_{\sigma} c_{\sigma} \neq 0
$$

and $c_{\sigma} \neq 0$ if $\sigma \neq \sigma_{0}$.

Introduce now a new function

$$
\Phi(z)=\left\{\begin{array}{lc}
\phi(z), & z \in D \\
\phi(T(z)) & z \in T(D) .
\end{array}\right.
$$

Extend next the definition of the function $\Phi(z)$ from the domain $D \cup T(D)$ into the region $\mathfrak{D}$ by the automorphicity law,

$$
\Phi(z)=\Phi\left(\sigma^{-1}(z)\right), \quad z \in \sigma(D \cup T(D)), \quad \sigma \in \mathfrak{G} \backslash \sigma_{0} .
$$

The function $\Phi(z)$ so defined is a piece-wise meromorphic function with the discontinuity line $\mathfrak{L}=\cup_{\sigma \in \mathfrak{G}} \sigma(L)$ invariant with respect to the group $\mathfrak{G}$ :

$$
\Phi(\sigma(z))=\Phi(z), \quad z \in \mathfrak{D} \backslash \mathfrak{L}, \quad \sigma \in \mathfrak{G} .
$$

In addition, the function $\Phi(z)$ satisfies the symmetry condition

$$
\overline{\Phi\left(T_{\nu}(z)\right)}=\Phi(z), \quad z \in \mathfrak{D} \backslash \mathfrak{L}, \quad \nu=0,1, \ldots, N,
$$

which follows from (2.6) and (2.7). In order to write the boundary condition (2.2) in terms of the function $\Phi(z)$, let $z \rightarrow t \in L_{\nu}, z \in D$. Then $T_{\nu}(z) \rightarrow t, T_{\nu}(z) \in T_{\nu}(D)$. Introduce the following notations

$$
\begin{aligned}
& \Phi^{+}(t)=\lim _{z \rightarrow t, z \in D} \Phi(z)=\phi(t), \\
& \Phi^{-}(t)=\lim _{z \rightarrow t, z \in T_{\nu}(D)} \Phi(z)=\lim _{T_{\nu}(z) \rightarrow t, T_{\nu}(z) \in D} \overline{\Phi\left(T_{\nu}(z)\right)}=\overline{\phi(t)} .
\end{aligned}
$$

Now inspection of the boundary condition (2.2) shows that it is essentially equivalent to the equation

$$
\operatorname{Re}\{[a(t)-i b(t)] \phi(t)\}=c(t), \quad t \in L \backslash \Theta,
$$

or, equivalently, in terms of the functions (2.10),

$$
\Phi^{+}(t)=p(t) \Phi^{-}(t)+q(t), \quad t \in L \backslash \Theta,
$$

where

$$
p(t)=-\frac{a(t)+i b(t)}{a(t)-i b(t)}, \quad q(t)=\frac{2 c(t)}{a(t)-i b(t)} .
$$

Definition 2.2. We say that a function $\Phi(z) \in Q_{\mathfrak{G}}(\mathfrak{L})$ if it is piece-wise meromorphic with the discontinuity line $\mathfrak{L}$, invariant with respect to the group $\mathfrak{G}: \Phi(\sigma(z))=\Phi(z)$, $\sigma \in \mathfrak{G}, z \in \mathfrak{D} \backslash \mathfrak{L}$, and $T$-symmetric: $\overline{\Phi(T(z))}=\Phi(z), z \in \mathfrak{D} \backslash \mathfrak{L}$.

The fact that the boundary values of the function $\Phi(z)$ satisfy the condition (2.12) lends itself to the opportunity of stating the following Riemann-Hilbert boundary-value problem with discontinuous coefficients in the class of functions $Q_{\mathfrak{G}}(\mathfrak{L})$ : 
Problem 2.3. Find all functions $\Phi(z) \in Q_{\mathfrak{G}}(\mathfrak{L})$, Hölder-continuous in the domain $\mathfrak{D} \cup$ $\mathfrak{L}$ apart from the set of points $\sigma(\Theta), \sigma \in \mathfrak{G}$, where they may have integrable singularities, bounded at the points $\sigma(\infty)$ and which satisfy the boundary condition (2.12).

3. Multiplicative canonical function. Let $t_{\nu 1}$ be the starting point of the circle $L_{\nu}$. This point can be chosen arbitrarily if both of the functions $a(t)$ and $b(t)$ are continuous everywhere on the circle $L_{\nu}$.

DEFINITION 3.1. We say that a function $\chi(z)$ is a multiplicative canonical function of Problem 2.3 if:

(i) it is a piece-wise meromorphic function in the region $\mathfrak{D}$ with the discontinuity line $\mathfrak{L}$, Hölder-continuous in the domain $\mathfrak{D} \cup \mathfrak{L}$ except for the points $\sigma\left(t_{\nu 1}\right)$, where it may have a power singularity of any finite exponent, and the points $\sigma\left(t_{\nu 2}\right), \sigma\left(t_{\nu 3}\right), \ldots, \sigma\left(t_{\nu m_{\nu}}\right)$, $\sigma \in \mathfrak{G}$, where it may have integrable singularities,

(ii) its boundary values $\chi^{ \pm}(t)$ satisfy the boundary condition

$$
\chi^{+}(t)=p(t) \chi^{-}(t), \quad t \in L \backslash \Theta,
$$

(iii) it is a $T$-symmetric function: $\chi(z)=\overline{\chi(T(z))}, z \in \mathfrak{D} \backslash \mathfrak{L}$, and

(iv) it satisfies the multiplicativity condition $\chi(\sigma(z))=H_{\sigma}^{-1} \chi(z), \sigma \in \mathfrak{G}, z \in \mathfrak{D} \backslash \mathfrak{L}$, with the character $H^{-1}$, where $H$ is a group homomorphism between $\mathfrak{G}$ and a multiplicative group $\mathfrak{H}$ of complex numbers such that $H_{\sigma \omega}=H_{\sigma} H_{\omega}$.

To find such a function, we shall use a quasiautomorphic analogue of the Cauchy kernel. Prove first its existence.

Theorem 3.2. There exists a function $K(z, \tau)$ which has the following properties:

(i) for each fixed $\tau \in L, K(z, \tau)=\frac{1}{\tau-z}+B(z, \tau)$, where $B(z, \tau)$ is an analytic function of $z \in \mathfrak{F}$,

(ii) there exists a point $z_{*} \in \mathfrak{F}$ such that $K\left(z_{*}, \tau\right)=0$ for all $\tau \in L$,

(iii) for any $\sigma \in \mathfrak{G}$,

$$
K(\sigma(z), \tau)=K(z, \tau)+\eta_{\sigma}(\tau)
$$

where $\eta_{\sigma}(\tau)=K\left(\sigma\left(z_{*}\right), \tau\right)$.

Proof. The existence of such a function for any discrete discontinuous group of Möbius transformations and, in particular, for a Schottky symmetry group, follows from the theory of abelian integrals on closed Riemann surfaces [18. Indeed, the fundamental region $\mathfrak{F}$ becomes a closed Riemann surface of genus $N$ if we add the circles $L_{\nu}^{\prime}=\sigma_{\nu}^{-1}\left(L_{\nu}\right)$ and consider all congruent points of the circles $L_{\nu}$ and $L_{\nu}^{\prime}(\nu=1,2, \ldots, N)$ as identical. The cycles $L_{\nu}$ may be accepted as canonical cross-sections $\mathbf{a}_{\nu}$, and any simple curve joining a pair of congruent points $\xi_{\nu}^{\prime} \in L_{\nu}^{\prime}$ and $\sigma_{\nu}\left(\xi_{\nu}^{\prime}\right) \in L_{\nu}$ can be considered as a canonical cross-section $\mathbf{b}_{\nu}$. As a function of $z$, the kernel $K(z, \tau)$ is an abelian integral of the second kind. It has only one simple pole at the point $z=\tau$ with the residue -1 . It vanishes at the point $z=z_{*}$ and has zero $A$-periods, $\oint_{a_{\nu}} d_{z} K(z, \tau)=0$. It is known [18] that such an abelian integral exists and it is unique. This integral has non-zero 
$B$-periods,

$$
\oint_{b_{\nu}} d_{z} K(z, \tau)=K\left(\sigma_{\nu}\left(\xi_{\nu}^{\prime}\right), \tau\right)-K\left(\xi_{\nu}^{\prime}, \tau\right)=\eta_{\nu}(\tau), \quad \nu=1,2, \ldots, N,
$$

where $\eta_{\nu}(\tau)=K\left(\sigma_{\nu}\left(z_{*}\right), \tau\right)$, and the maps $\sigma_{\nu}=T_{\nu} T$ are generating transformations of the group $\mathfrak{G}$.

Note that the functions $\eta_{\nu}(\tau)(\nu=1,2, \ldots, N)$ are linearly independent, and the differentials $\frac{1}{2 \pi i} \eta_{\nu}(\tau) d \tau(\nu=1,2, \ldots, N)$ form the normalized basis of abelian differentials of the first kind on the Riemann surface,

$$
\begin{aligned}
& \frac{1}{2 \pi i} \int_{L_{\nu}} \eta_{k}(\tau) d \tau=\left\{\begin{array}{l}
1, k=\nu, \\
0, k \neq \nu,
\end{array}\right. \\
& \frac{1}{2 \pi i} \int_{T\left(t_{\nu 1}\right)}^{t_{\nu 1}} \eta_{k}(\tau) d \tau=B_{k \nu} .
\end{aligned}
$$

The matrix of $B$-periods, $\left\|B_{k \nu}\right\|, k, \nu=1,2, \ldots, N$, is symmetric and its imaginary part is positive definite.

Definition 3.3. A function $K(z, \xi)$ is said to be a quasiautomorphic analogue of the Cauchy kernel if it possesses properties (i) to (iii) of Theorem 3.2

REMARK 3.4. Because of the property (i), the integral

$$
\frac{1}{2 \pi i} \int_{L} \varphi(\tau) K(z, \tau) d \tau
$$

satisfies the Sokhotski-Plemelj formulas.

If the group $\mathfrak{G}$ is of the first class [7] or, equivalently, if the numerical series

$$
\sum_{\sigma \in \mathfrak{G} \backslash \sigma_{0}} \frac{\left|a_{\sigma} d_{\sigma}-b_{\sigma} c_{\sigma}\right|}{\left|c_{\sigma}\right|^{2}}
$$

is convergent, such a kernel is known [8],

$$
K(z, \tau)=\sum_{\sigma \in \mathfrak{G}}\left(\frac{1}{\sigma(\tau)-z}-\frac{1}{\sigma(\tau)-z_{*}}\right) \sigma^{\prime}(\tau)=\sum_{\sigma \in \mathfrak{G}}\left(\frac{1}{\tau-\sigma(z)}-\frac{1}{\tau-\sigma\left(z_{*}\right)}\right) .
$$

In general, the kernel can be expressed through the Schottky-Klein prime function $\omega(z, \tau)$ associated with the group $\mathfrak{G}[\underline{6}$ by the formula

$$
K(z, \tau)=\frac{d}{d \tau} \ln \left(\frac{\omega(z, \tau)}{\omega\left(z_{*}, \tau\right)}\right) .
$$

Define next the logarithmic function $\ln p(\tau)$ or, equivalently, $\arg p(\tau)$ on each arc $t_{\nu j} t_{\nu j+1}, \nu=0,1, \ldots, N, j=1,2, \ldots m_{\nu}$. We shall use the definitions $t_{\nu j}^{+}$and $t_{\nu j+1}^{-}$ to indicate the starting and the terminal points of the arc $t_{\nu j} t_{\nu j+1}$, respectively (it is assumed that $t_{\nu m_{\nu}+1}=t_{\nu 1}$ ). On the $\operatorname{arc} t_{\nu 1} t_{\nu 2}$, a branch of the function $\arg p(\tau)$ can be fixed arbitrarily. We fix it by the condition

$$
-\pi<\arg p\left(t_{\nu 1}^{+}\right) \leq \pi
$$


Let $\Delta_{\nu j}$ be the change of $\arg p(\tau)$ along the $\operatorname{arc} t_{\nu j} t_{\nu j+1}\left(j=1,2, \ldots, m_{\nu}\right)$,

$$
\Delta_{\nu j}=[\arg p(\tau)]_{t_{\nu j} t_{\nu j+1}} .
$$

Then, obviously, $\arg p\left(t_{\nu j+1}^{-}\right)=\arg p\left(t_{\nu j}^{+}\right)+\Delta_{\nu j}, j=1,2, \ldots, m_{\nu}$. The values $\arg p\left(t_{\nu j}^{+}\right)$ $\left(j=2,3, \ldots, m_{\nu}\right)$ cannot be chosen arbitrarily. Since the solution $\Phi(z)$ may have integrable singularities at the points $t_{\nu j}$, define a continuous branch of the function $\arg p(\tau)$ by

$$
-2 \pi<\arg p\left(t_{\nu j}^{-}\right)-\arg p\left(t_{\nu j}^{+}\right) \leq 0, \quad j=2, \ldots, m_{\nu}
$$

We next choose integers $\kappa_{\nu}$ such that

$$
-4 \pi<\arg p\left(t_{\nu 1}^{-}\right)-\arg p\left(t_{\nu 1}^{+}\right)-4 \pi \kappa_{\nu} \leq 0, \quad \nu=0,1, \ldots, N,
$$

and prove the following result.

Theorem 3.5. Let

$$
\Gamma(z)=\frac{1}{4 \pi} \int_{L} \arg p(\tau) K(z, \tau) d \tau+\sum_{\nu=0}^{N} \operatorname{sgn} \kappa_{\nu} \sum_{j=1}^{\left|\kappa_{\nu}\right|} \int_{\gamma_{\nu j}} K(z, \tau) d \tau,
$$

where $\gamma_{\nu j}=t_{\nu 1} z_{\nu j}$ are piece-wise smooth curves in $D$ which do not cross each other, and $z_{\nu j}$ are arbitrarily fixed distinct points in the region $D$ (Fig. 1). Then

$$
\chi(z)=\exp \{\Gamma(z)+\overline{\Gamma(T(z))}\}
$$

is a multiplicative canonical function.

Proof. Analyze first the behavior of the function $\chi(z)$ at the points of the set $\Theta$. Clearly, in a neighborhood of the point $z=t_{\nu j}$,

$$
\Gamma(z)=\alpha_{\nu j} \ln \left(z-t_{\nu j}\right)+f_{0}(z), \quad j=1,2, \ldots, m_{\nu}, \quad \nu=0,1, \ldots, N,
$$

where $f_{0}(z)$ is a function bounded as $z \rightarrow t_{\nu j}$,

$$
\begin{aligned}
& \alpha_{\nu 1}=\frac{1}{4 \pi}\left[\arg p\left(t_{\nu 1}^{-}\right)-\arg p\left(t_{\nu 1}^{+}\right)\right]-\kappa_{\nu}, \\
& \alpha_{\nu j}=\frac{1}{4 \pi}\left[\arg p\left(t_{\nu j}^{-}\right)-\arg p\left(t_{\nu j}^{+}\right)\right], \quad j=2,3, \ldots, m_{\nu} .
\end{aligned}
$$

Since $T_{\nu}\left(t_{\nu j}\right)=t_{\nu j}$, we conclude from (2.3) that

$$
T_{\nu}(z)-t_{\nu j} \sim-\frac{\rho_{\nu}^{2}}{\left(\bar{t}_{\nu j}-\bar{\delta}_{\nu}\right)^{2}}\left(\bar{z}-\bar{t}_{\nu j}\right), \quad z \rightarrow t_{\nu j} .
$$

On the other hand,

$$
\begin{aligned}
\Gamma(T(z)) & =\Gamma\left(\sigma_{\nu}^{-1}\left(T_{\nu}(z)\right)\right)=\Gamma\left(T_{\nu}(z)\right)-\Gamma\left(\sigma_{\nu}\left(z_{*}\right)\right) \\
& =\alpha_{\nu j} \ln \left(T_{\nu}(z)-t_{\nu j}\right)+f_{1}(z), \quad z \rightarrow t_{\nu j},
\end{aligned}
$$

where $f_{1}(z)$ is a function bounded as $z \rightarrow t_{\nu j}$. From the definition of the function $\chi(z)$ (3.14) and (3.13), it follows that

$$
\begin{aligned}
\chi(z) \sim A_{\nu j}\left(z-t_{\nu j}\right)^{2 \alpha_{\nu j}}, \quad A_{\nu j} & =\text { const } \neq 0, \quad z \rightarrow t_{\nu j}, \\
j=1,2, \ldots, m_{\nu}, \quad \nu & =0,1, \ldots, N .
\end{aligned}
$$


Since $-\frac{1}{2}<\alpha_{\nu j} \leq 0(j \neq 1)$, the function $\chi(z)$ may have at most an integrable singularity as $z \rightarrow t_{\nu j}(j \neq 1)$. At the point $z=t_{\nu 1}$, the function $\chi(z)$ has an integrable singularity if $-\frac{1}{2}<\alpha_{\nu 1} \leq 0$ and a nonintegrable singularity of order $1 \leq-2 \alpha_{\nu 1}<2$ if $-1<\alpha_{\nu 1} \leq-\frac{1}{2}$.

Analysis of the second term in (3.13) implies that if $\kappa_{\nu} \neq 0$, then at the points $z_{\nu j}$, the function $\chi(z)$ has a simple pole provided $\kappa_{\nu}$ is negative and a simple zero provided $\kappa_{\nu}$ is positive. Apart from these points, the function $\chi(z)$ is analytic everywhere in the region $D$ and does not vanish. In the case $\kappa_{\nu}=0, z_{\nu j}$ are regular points of the function $\chi(z)$.

Verify now that the boundary values, $\chi^{+}(t)$ and $\chi^{-}(t)$, of the function $\chi(z)$ satisfy the linear relation (3.1). By applying the Sokhotski-Plemelj formulas to the integral

$$
\Gamma_{0}(z)=\frac{1}{4 \pi i} \int_{L} \ln p(\tau) K(z, \tau) d \tau,
$$

and noticing that $|p(\tau)|=1$, we obtain

$$
\Gamma_{0}^{+}(t)-\Gamma_{0}^{-}(t)=\frac{i}{2} \arg p(t), \quad t \in L \backslash \Theta .
$$

Consider now $\Gamma_{0}^{ \pm}(T(t)), t \in L$. Let first $t \in L_{0}$ and $z \rightarrow t^{ \pm}$. Clearly then, $T(z) \rightarrow t^{\mp}$ and $\Gamma_{0}^{ \pm}(T(t))=\Gamma_{0}^{\mp}(t)$. This implies

$$
\Gamma_{0}^{+}(T(t))-\Gamma_{0}^{-}(T(t))=-\frac{i}{2} \arg p(t)
$$

For $t \in L_{\nu}(\nu=1,2, \ldots, N)$, because of the identity

$$
\Gamma_{0}(T(z))=\Gamma_{0}\left(T_{\nu}(z)\right)-\Gamma_{0}\left(\sigma_{\nu}\left(z_{*}\right)\right)
$$

the one-sided limits $\Gamma_{0}^{ \pm}\left(T_{0}(t)\right)$ of the function $\Gamma_{0}(T(z))$ meet the condition (3.22). Therefore, the jump of the function $\Gamma_{0}(z)+\overline{\Gamma_{0}(T(z))}$, when $z$ passes through the line $L$, equals $i \arg p(t)=\ln p(t)$.

Notice that the function $\Gamma(z)$ is discontinuous when $z$ passes through the curves $t_{\nu 1} z_{\nu j}$, and the jump is a multiple of $2 \pi i$. This means that the function $\chi(z)$ itself is continuous through these curves.

We observe next that the function $\chi(z)$ is a $T$-symmetric function: $\overline{\chi(T(z))}=\chi(z)$, $z \in \mathfrak{D} \backslash \mathfrak{L}$. To finalize the proof of the theorem, we need to show that $\chi(z)$ is a multiplicative function. The property (3.2) of the kernel $K(z, \tau)$ written for the generating transformations $\sigma_{\nu}(z)$ implies

$$
\begin{aligned}
\Gamma\left(\sigma_{\nu}(z)\right) & =\Gamma(z)+h_{\nu} \\
\Gamma\left(T\left(\sigma_{\nu}(z)\right)\right)=\Gamma\left(T T_{\nu} T(z)\right) & =\Gamma\left(\sigma_{\nu}^{-1} T(z)\right)=\Gamma(T(z))-h_{\nu},
\end{aligned}
$$

where $h_{\nu}=\Gamma\left(\sigma_{\nu}\left(z_{*}\right)\right), \nu=1,2 \ldots, N$. Therefore,

$$
\begin{gathered}
\chi\left(\sigma_{\nu}(z)\right)=H_{\nu}^{-1} \chi(z), \quad z \in \mathfrak{D} \backslash \mathfrak{L}, \\
H_{\nu}=\exp \left(-2 i \operatorname{Im} h_{\nu}\right), \quad \nu=1,2, \ldots, N .
\end{gathered}
$$

Consider now the general form of the transformation $\sigma=T_{\nu_{2 \mu}} T_{\nu_{2 \mu-1}} \ldots T_{\nu_{2}} T_{\nu_{1}}$. It can also be written in the form

$$
\sigma=\sigma_{\nu_{2 \mu}} \sigma_{\nu_{2 \mu-1}}^{-1} \ldots \sigma_{\nu_{2}} \sigma_{\nu_{1}}^{-1}, \quad \mu=1,2, \ldots
$$


Since

$$
\chi\left(\sigma_{\nu}^{-1}(z)\right)=H_{\nu} \chi(z), \quad z \in \mathfrak{D} \backslash \mathfrak{L},
$$

the property (3.25) for the generating transformations is valid for any transformation $\sigma \in \mathfrak{G}$ provided the number $H_{\nu}$ is replaced by $H_{\sigma}$,

$$
\begin{aligned}
\chi(\sigma(z)) & =H_{\sigma}^{-1} \chi(z), \quad z \in \mathfrak{D} \backslash \mathfrak{L}, \\
H_{\sigma} & =\frac{H_{\nu_{2}} H_{\nu_{4}} \ldots H_{\nu_{2 \mu}}}{H_{\nu_{1}} H_{\nu_{3}} \ldots H_{\nu_{2 \mu-1}}}, \quad H_{\sigma_{0}}=1 .
\end{aligned}
$$

The one-to-one map (3.28), $H$, from the group $\mathfrak{G}$ into a multiplicative group $\mathfrak{H}$ of complex numbers $H_{\sigma}, \sigma \in \mathfrak{G}$, has the following property:

$$
H_{\sigma \omega}=H_{\sigma} H_{\omega} \quad \forall \sigma, \omega \in \mathfrak{G} .
$$

Thus, $H$ is a homomorphism between these two groups, and $\chi(z)$ is a multiplicative function with the character $H^{-1}$ [19].

4. Quasimultiplicative analogue of the Cauchy kernel. To solve the homogeneous case $(q(t) \equiv 0)$ of Problem $[2.3$, we need a quasimultiplicative analogue of the Cauchy kernel.

Theorem 4.1. There exists a function $M(z, \tau)$ which has the following properties:

(i) for each fixed $\tau \in L, M(z, \tau)=\frac{1}{\tau-z}+B_{0}(z, \tau)$, where $B_{0}(z, \tau)$ is an analytic function of $z \in \mathfrak{F}$,

(ii) there exists a point $z_{0} \in \mathfrak{F}$ such that $M\left(z_{0}, \tau\right)=0$ for all $\tau \in L$,

(iii) for each fixed $\tau \in L$ and for any $\sigma \in \mathfrak{G}$, there exists a function $\zeta_{\sigma}(\tau)$ such that

$$
M(\sigma(z), \tau)=H_{\sigma} M(z, \tau)+\zeta_{\sigma}(\tau) .
$$

Definition 4.2. A function $M(z, \xi)$ which possesses properties (i) to (iii) is said to be a quasimultiplicative analogue of the Cauchy kernel with the character $H$.

Proof. Introduce a function $P(z)=\frac{\partial M(z, \tau)}{\partial z}$. From the conditions (i) and (iii), it follows that

$$
P(z)=\frac{1}{(\tau-z)^{2}}+B_{1}(z, \tau) \text { for each fixed } \tau \in L,
$$

where $B_{1}(z, \tau)=\frac{\partial B_{0}(z, \tau)}{\partial z}$, and also

$$
\sigma^{\prime}(z) P(\sigma(z))=H_{\sigma} P(z), \quad \sigma \in \mathfrak{G},
$$

or, equivalently, $\sigma_{\nu}^{\prime}(z) P\left(\sigma_{\nu}(z)\right)=H_{\nu} P(z), \nu=1,2, \ldots, N$. This means that $P(z)$ is a multiplicative automorphic form of weight (dimension) (-2) belonging to the character $H$ [19. This form has only one singularity in the fundamental region $\mathfrak{F}$, a pole of the second order at the point $z=\tau$. At the infinite point, $z=\infty$, it has a zero of the second order. In what follows we prove that such a form exists.

Let $\mathfrak{R}$ be a Riemann surface formed by gluing the congruent sides $L_{\nu}$ and $L_{\nu}^{\prime}=$ $\sigma_{\nu}^{-1}\left(L_{\nu}\right)$ of the fundamental region $\mathfrak{F}$. Choose the canonical cross-sections of the surface $\mathfrak{R}$ (the canonical homology basis on $\mathfrak{R}$ ) as follows: $\mathbf{a}_{\nu}=L_{\nu}$ and $\mathbf{b}_{\nu}=\xi_{\nu}^{\prime} \xi_{\nu}$ with $\xi_{\nu}^{\prime} \in L_{\nu}^{\prime}$ 
and $\xi_{\nu}=\sigma_{\nu}\left(\xi_{\nu}^{\prime}\right) \in L_{\nu}$. On the surface $\mathfrak{R}$, the differential $d P^{\circ}(z)=P(z) d z$ can be interpreted as a multiplicative differential with the character $H$ defined by

$$
H\left[\mathbf{a}_{\nu}\right]=1, \quad H\left[\mathbf{b}_{\nu}\right]=H_{\nu}, \quad \nu=1,2, \ldots, N .
$$

Show next that there exist exactly $N+1$ linearly independent multiplicative differentials which, on the surface $\mathfrak{R}$, have only one pole of multiplicity not higher than 2 . Let $r_{*}$ be the dimension of the space $\mathfrak{M}_{H}\left(\mathfrak{d}_{*}\right)$ of multiplicative differentials with the character $H$ whose divisors $\mathfrak{d}_{*}$ are multiples of the divisor $\mathfrak{d}_{0}=\tau^{-2}$, and let $r$ be the dimension of the space $\mathfrak{M}_{H^{-1}}\left(\mathfrak{d}_{*}^{-1}\right)$ of multiplicative functions with the character $H^{-1}$ whose divisors $\mathfrak{d}_{*}^{-1}$ are multiples of the divisor $\mathfrak{d}_{0}^{-1}=\tau^{2}$. The character $H^{-1}$ is defined by the factors $H^{-1}\left[\mathbf{a}_{\nu}\right]=1, H^{-1}\left[\mathbf{b}_{\nu}\right]=H_{\nu}^{-1}, \nu=1,2, \ldots, N$. The space $\mathfrak{M}_{H^{-1}}\left(\mathfrak{d}_{*}^{-1}\right)$ consists of multiplicative functions which have a second-order zero at the point $z=\tau$ and which do not have any singularities on $\mathfrak{R}$. Clearly, the dimension of this space, $r$, is zero. By the Riemann-Roch theorem for multiplicative functions on a genus- $N$ Riemann surface $\Re$ [13], p. 126 ,

$$
r_{*}=r-\operatorname{deg} \mathfrak{d}_{0}+N-1 \text {. }
$$

Since $r=0$ and $\operatorname{deg} \mathfrak{d}_{0}=-2$ we find $r_{*}=N+1$. Similarly, the dimension of the space of multiplicative differentials whose divisors are multiples of the divisor $\mathfrak{d}_{1}=\tau^{-1}$ equals $N$. Note, however, that there does not exist a differential $d P^{\circ}$ with factors $H\left[\mathbf{a}_{\nu}\right]=1$ analytic everywhere on the surface $\mathfrak{R}$ except at the point $z=\tau$,

$$
d P^{\circ}(z) \sim \frac{d z}{\tau-z} .
$$

Otherwise, on one hand,

$$
\int_{\partial \mathfrak{F}} d P^{\circ}=-2 \pi i
$$

On the other hand,

$$
\int_{\partial \mathfrak{F}} d P^{\circ}=\sum_{\nu=1}^{N}\left(\int_{L_{\nu}} d P^{\circ}-\int_{L_{\nu}^{\prime}} d P^{\circ}\right)=\sum_{\nu=1}^{N}\left(1-H^{-1}\left[\mathbf{b}_{\nu}\right]\right) \int_{L_{\nu}} d P^{\circ}=0
$$

since

$$
\int_{L_{\nu}} d P^{\circ}=P^{\circ}\left(\xi_{\nu}^{-}\right)-P^{\circ}\left(\xi_{\nu}^{+}\right)=\left(H\left[\mathbf{a}_{\nu}\right]-1\right) P^{\circ}\left(\xi_{\nu}^{+}\right)=0 ;
$$

that is in contradiction with (4.7). This means there exist $N$ linearly independent multiplicative differentials with factors $H\left[\mathbf{a}_{\nu}\right]=1$ and $H\left[\mathbf{b}_{\nu}\right]=H_{\nu}, \nu=1,2, \ldots, N$, analytic everywhere on the surface $\mathfrak{R}$. Since $r_{*}=N+1$, there exists at least one multiplicative differential $d P^{\circ}(z)$ with factors $H\left[\mathbf{a}_{\nu}\right]=1$ and $H\left[\mathbf{b}_{\nu}\right]=H_{\nu}$ and which has a single second-order pole with the principal part $\frac{d z}{(\tau-z)^{2}}$. Then the function $P(z)=\frac{d P^{\circ}(z)}{d z}$ satisfies the conditions (4.2) and (4.3), has a second-order zero at the infinite point $z=\infty$, and also has the following property:

$$
\int_{L_{\nu}} P(z) d z=\int_{L_{\nu}} d P^{\circ}=0 .
$$


Define now a function

$$
M(z, \tau)=\int_{z_{0}}^{z} P(\xi) d \xi
$$

This function, as a function of $z$ and for any fixed $\tau \in L$, is analytic everywhere in the region $\mathfrak{F}$ except at the point $z=\tau$, where it has a simple pole with the residue -1 . Clearly, $M\left(z_{0}, \tau\right)=0$. Finally, from (4.3),

$$
\begin{aligned}
M(\sigma(z), \tau) & =\int_{z_{0}}^{\sigma\left(z_{0}\right)} P(\xi) d \xi+\int_{\sigma\left(z_{0}\right)}^{\sigma(z)} P(\xi) d \xi \\
& =M\left(\sigma\left(z_{0}\right), \tau\right)+\int_{z_{0}}^{z} P(\sigma(\xi)) d \sigma(\xi)=H_{\sigma} M(z, \tau)+\zeta_{\sigma}(\tau), \quad \sigma \in \mathfrak{G}
\end{aligned}
$$

where $\zeta_{\sigma}(\tau)=M\left(\sigma\left(z_{0}\right), \tau\right)$. Because of the condition (4.10), $M(z, \tau)$ is a single-valued function in the fundamental region $\mathfrak{F}$. The quasimultiplicativity property (4.12) implies that the function $M(z, \tau)$ is single-valued everywhere in the region $\mathfrak{D}$. Thus, all three properties (i) to (iii) have been verified, and that proves the existence of the kernel $M(z, \tau)$.

If the group $\mathfrak{G}$ is of the first class, and $\left|H_{\sigma}\right|=1$ for all $\sigma \in \mathfrak{G}$ or, equivalently, $\left|H_{\nu}\right|=1$, $\nu=1,2, \ldots, N$, then the kernel $M(z, \tau)$ can be found explicitly [20] through all the transformations of the group $\mathfrak{G}$ in the form of an absolutely and uniformly convergent series

$$
M(z, \tau)=\sum_{\sigma \in \mathfrak{G}} \frac{1}{H_{\sigma}}\left(\frac{1}{\tau-\sigma(z)}-\frac{1}{\tau-\sigma\left(z_{0}\right)}\right) .
$$

This kernel vanishes at $z=z_{0}$ and possesses the other two properties of the kernel $M(z, \tau)$. Indeed, because $\mathfrak{G} \ni \sigma_{0}$,

$$
M(z, \tau)=\frac{1}{\tau-z}-\frac{1}{\tau-z_{0}}+\sum_{\sigma \in \mathfrak{G} \backslash \sigma_{0}} \frac{1}{H_{\sigma}}\left(\frac{1}{\tau-\sigma(z)}-\frac{1}{\tau-\sigma\left(z_{0}\right)}\right) .
$$

To verify the property (4.1), we employ the multiplicativity of $H, H_{\sigma \omega}=H_{\sigma} H_{\omega}$, the relation

$$
\begin{aligned}
M(\sigma(z), \tau)= & \sum_{\omega \in \mathfrak{G}} \frac{1}{H_{\omega}}\left(\frac{1}{\tau-\omega \sigma(z)}-\frac{1}{\tau-\omega \sigma\left(z_{0}\right)}\right) \\
& +\sum_{\omega \in \mathfrak{G}} \frac{1}{H_{\omega}}\left(\frac{1}{\tau-\omega \sigma\left(z_{0}\right)}-\frac{1}{\tau-\omega\left(z_{0}\right)}\right),
\end{aligned}
$$

and make the substitution $\nu=\omega \sigma$. This ultimately gives the relation wanted: $M(\sigma(z), \tau)$ $=H_{\sigma} M(z, \tau)+\zeta_{\sigma}(\tau)$, where $\zeta_{\sigma}(\tau)=M\left(\sigma\left(z_{0}\right), \tau\right)$.

5. Solution to the Riemann-Hilbert problem. Being now equipped with two analogues of the Cauchy kernel, the quasiautomorphic and quasimultiplicative kernels $K(z, \tau)$ and $M(z, \tau)$, we solve Problem 2.3. We begin with the homogeneous RiemannHilbert problem. 
5.1. Homogeneous case: $q(t) \equiv 0$.

Problem 5.1. Find all functions $\Phi(z) \in Q_{\mathfrak{G}}(\mathfrak{L})$, Hölder-continuous everywhere in the domain $\mathfrak{D} \cup \mathfrak{L}$ apart from the set of points $\sigma(\Theta), \sigma \in \mathfrak{G}$, where they may have integrable singularities, bounded at the points $\sigma(\infty)$ and satisfying the boundary condition

$$
\Phi^{+}(t)=p(t) \Phi^{-}(t), \quad t \in L \backslash \Theta .
$$

By using (3.1), we write the boundary condition (5.1) in the form

$$
\frac{\Phi^{+}(t)}{\chi^{+}(t)}=\frac{\Phi^{-}(t)}{\chi^{-}(t)}, \quad t \in L \backslash \Theta
$$

and analyze the function $\Phi(z) / \chi(z)$. It is a $T$-symmetric and $\mathfrak{G}$-multiplicative function with the factors $H_{\sigma}, \sigma \in \mathfrak{G}$. If $\kappa_{\nu}>0$, then it has simple poles at the points $z_{\nu j}$ and $T\left(z_{\nu j}\right)\left(j=1,2, \ldots, m_{\nu}\right)$. In the case $\kappa_{\nu}<0$, the function $\Phi(z) / \chi(z)$ has simple zeros at the points $z_{\nu j}$ and $T\left(z_{\nu j}\right)$. Let

$$
\kappa_{\nu}^{+}=\left\{\begin{array}{c}
\kappa_{\nu}, \kappa_{\nu}>0, \\
0, \quad \kappa_{\nu} \leq 0,
\end{array} \kappa_{\nu}^{-}=\left\{\begin{array}{c}
0, \quad \kappa_{\nu} \geq 0 \\
\kappa_{\nu}, \kappa_{\nu}<0
\end{array}\right.\right.
$$

and

$$
\kappa^{+}=\sum_{\nu=0}^{N} \kappa_{\nu}^{+}, \quad \kappa^{-}=\sum_{\nu=0}^{N} \kappa_{\nu}^{-}
$$

For arbitrary complex numbers $C_{\nu j}$, we define

$$
\Omega_{0}(z)=\sum_{\nu=0}^{N} \sum_{j=1}^{\kappa_{\nu}^{+}} C_{\nu j} M\left(z, z_{\nu j}\right)
$$

Since the function $M(z, \hat{z})$ has a single pole $z=\hat{z} \in \mathfrak{F}$, it is quite clear that the functions $\Omega_{0}(z)+\overline{\Omega_{0}(T(z))}$ and $\Phi(z) / \chi(z)$ have simple poles at the same points of the region $\mathfrak{F}$. By the generalized Liouville theorem for multiplicative functions,

$$
\Phi(z)=\chi(z)\left[C_{0}+\Omega_{0}(z)+\overline{\Omega_{0}(T(z))}\right]
$$

where $C_{0}$ is a constant.

For arbitrary constants $C_{0}$ and $C_{\nu j}$, the function (5.6) cannot be accepted as the solution to Problem 5.1. Indeed, it must be $T$-symmetric, $\mathfrak{G}$-automorphic and piece-wise meromorphic. This is guaranteed if the constant $C_{0}$ is real and the function $\Phi(z) / \chi(z)$ is multiplicative with factors $H_{\nu}$. Because of the quasimultiplicativity property (4.1) of the kernel $M(z, \tau)$, however, in general,

$$
\frac{\Phi\left(\sigma_{k}(z)\right)}{\chi\left(\sigma_{k}(z)\right)}=H_{k} \frac{\Phi(z)}{\chi(z)}+\xi_{k}, \quad k=1,2, \ldots, N
$$

where

$$
\xi_{k}=C_{0}\left(1-H_{k}\right)+\sum_{\nu=0}^{N} \sum_{j=1}^{\kappa_{\nu}^{+}}\left[C_{\nu j} \zeta_{k}\left(z_{\nu j}\right)-H_{k} \overline{C_{\nu j} \zeta_{k}\left(z_{\nu j}\right)}\right]
$$

where $\zeta_{k}(z)=M\left(\sigma_{k}\left(z_{0}\right), z\right)$. Thus, the function $\Phi(z) / \chi(z)$ becomes multiplicative if and only if $\xi_{k}=0$. This condition can be written as follows:

$$
\operatorname{Im}\left\{H_{k}^{-1 / 2} C_{0}+H_{k}^{-1 / 2} \Omega_{0}\left(\sigma_{k}\left(z_{0}\right)\right)\right\}=0, \quad k=1,2, \ldots, N .
$$


Next, for negative $\kappa_{\nu}$, the function (5.6) has simple poles at the points $z_{\nu j}$. These points become removable points if the following conditions are met:

$$
C_{0}+\Omega_{0}\left(z_{\nu j}\right)+\overline{\Omega_{0}\left(T\left(z_{\nu j}\right)\right)}=0, \quad j=1,2, \ldots,-\kappa_{\nu}^{-}, \quad \nu=0,1, \ldots, N .
$$

Finally, if $-1<\alpha_{\nu 1} \leq-\frac{1}{2}$, then the function (5.6) has a nonintegrable singularity at the point $t_{\nu 1}$. To make this singularity integrable we require

$$
l_{\nu}\left[C_{0}+\Omega_{0}\left(t_{\nu 1}\right)+\overline{\Omega_{0}\left(T\left(t_{\nu 1}\right)\right)}\right]=0, \quad \nu=0,1, \ldots, N,
$$

where

$$
l_{\nu}=\left\{\begin{array}{l}
0, \quad-1 / 2<\alpha_{\nu 1} \leq 0 \\
1,-1<\alpha_{\nu 1} \leq-1 / 2
\end{array}\right.
$$

The condition (5.11) can be simplified. We consider two cases. If $t_{\nu 1}=t_{01}$, then $T\left(t_{01}\right)=t_{01}$, and

$$
C_{0}+\Omega_{0}\left(t_{01}\right)+\overline{\Omega_{0}\left(T\left(t_{01}\right)\right)}=C_{0}+2 \operatorname{Re} \Omega_{0}\left(t_{01}\right) .
$$

If $\nu \neq 0$, then

$$
\overline{\Omega_{0}\left(T\left(t_{\nu 1}\right)\right)}=\overline{\Omega_{0}\left(\sigma_{\nu}^{-1} T_{\nu}\left(t_{\nu 1}\right)\right)}=H_{\nu} \overline{\Omega_{0}\left(t_{\nu 1}\right)}-H_{\nu} \overline{\Omega_{0}\left(\sigma_{\nu}\left(z_{0}\right)\right)} .
$$

Then, by using the condition (5.9), it is easy to verify that

$$
C_{0}+\Omega_{0}\left(t_{\nu 1}\right)+\overline{\Omega_{0}\left(T\left(t_{\nu 1}\right)\right)}=H_{\nu}^{1 / 2} \operatorname{Re}\left\{H_{\nu}^{-1 / 2}\left[C_{0}+2 \Omega_{0}\left(t_{\nu 1}\right)-\Omega_{0}\left(\sigma_{\nu}\left(z_{0}\right)\right)\right]\right\} .
$$

The two formulas (5.13) and (5.15) can be combined, and the conditions (5.11) become

$$
l_{\nu} \operatorname{Re}\left\{H_{\nu}^{-1 / 2}\left[C_{0}+2 \Omega_{0}\left(t_{\nu 1}\right)-\Omega_{0}\left(\sigma_{\nu}\left(z_{0}\right)\right)\right]\right\}=0, \quad \nu=0,1, \ldots, N .
$$

Thus, there are $2 \kappa^{+}+1$ real arbitrary constants, $C_{0}$ and $C_{\nu j}=C_{\nu j}^{\prime}+i C_{\nu j}^{\prime \prime}$. These constants need to satisfy $N$ real conditions (5.9),,$-\kappa^{-}$complex conditions (5.10), and $l=\sum_{\nu=0}^{N} l_{\nu}$ real conditions (5.16), in total, $N+l-2 \kappa^{-}$real conditions.

Let $\kappa=\kappa^{+}+\kappa^{-}=\sum_{\nu=0}^{N} \kappa_{\nu}$. Introduce an integer

$$
\mathfrak{K}=2 \kappa-l=\sum_{\nu=0}^{N}\left(2 \kappa_{\nu}-l_{\nu}\right)
$$

and call this number the index of Problems 5.1 and 2.3. Denote by $\rho$ the rank of the linear system of $N+l-2 \kappa^{-}$real equations (5.9), (5.10), and (5.16) $\left(1 \leq \rho \leq N-2 \kappa^{-}+l\right)$.

Theorem 5.2. If the index $\mathfrak{K}$ is negative, then Problem 5.1 has only the trivial solution.

If $0 \leq \mathfrak{K} \leq 2 N-2$, then Problem 5.1 has $2 \kappa^{+}-\rho+1$ nontrivial solutions (5.6) over the field of real numbers, provided this number is positive, and only the trivial solution otherwise.

If $\mathfrak{K}>2 N-2$, then Problem 5.1 has $\mathfrak{K}-N+1$ solutions (5.6) over the field of real numbers.

Proof. The multiplicative function $\Phi(z) / \chi(z)$ has simple zeros at the points $z_{\nu j}$ and $T\left(z_{\nu j}\right)$ if $\kappa_{\nu}<0$ and at the points $t_{\nu 1}$ if $-1<\alpha_{\nu 1} \leq-\frac{1}{2}$. The number of these points is equal to $l-2 \kappa^{-}$. The function $\Phi(z) / \chi(z)$ may have some other zeros. That is why the number of zeros in the fundamental region is not less than $l-2 \kappa^{-}$. In the case $\kappa_{\nu}>0$, 
this function has $2 \kappa^{+}$simple poles at the points $z_{\nu j}$ and $T\left(z_{\nu j}\right)$. It is clear that the divisor of the multiplicative function $\Phi(z) / \chi(z)$ is a multiple of the divisor

$$
\mathfrak{d}=\prod_{\nu=0}^{N} t_{\nu 1}^{l_{\nu}} \prod_{j=1}^{\left|\kappa_{\nu}\right|} z_{\nu j}^{-\kappa_{\nu}}\left[T\left(z_{\nu j}\right)\right]^{-\kappa_{\nu}},
$$

and $\operatorname{deg} \mathfrak{d}=-2 \kappa+l=-\mathfrak{K}$.

Let first $\mathfrak{K}<0$. Then the degree of the divisor $\mathfrak{d}$ is positive and since $\mathfrak{K}=2 \kappa^{+}-(l-$ $\left.2 \kappa^{-}\right)$, this implies that the number of zeros of the multiplicative function $\Phi(z) / \chi(z)$ in the fundamental region $\mathfrak{F}$ is greater than the number of poles. Such a nontrivial function does not exist.

Let now $\mathfrak{K}>2 N-2$. Notice that the dimension $d^{-}$of the space of multiplicative forms of weight (-2) with factors $H_{\sigma}^{-1}, \sigma \in \mathfrak{G}$, whose divisors are multiples of the divisor $\mathfrak{d}^{-1}$, is equal to zero. Indeed, on one hand, $\operatorname{deg} \mathfrak{d}^{-1}=\mathfrak{K}$ and $\mathfrak{K}>2 N-2$. On the other hand, the degree of the divisor of any multiplicative form of weight (-2) on a genus- $N$ Riemann surface is equal to $2 N-2$ [19]. Let $d^{+}$be the dimension of the space of multiplicative functions with factors $H_{\sigma}, \sigma \in \mathfrak{G}$, whose divisors are multiples of the divisor $\mathfrak{d}$. Then by the Riemann-Roch theorem [13,

$$
d^{+}=\operatorname{deg} \mathfrak{d}^{-1}-N+1+d^{-},
$$

and $d^{+}=\mathfrak{K}-N+1$. Since the solution $\Phi(z)$ has to be a $T$-symmetric function, Problem 5.1 has $\mathfrak{K}-N+1$ linearly independent solutions over the field of real numbers.

In the final case, $0 \leq \mathfrak{K} \leq 2 N-2$, the number of solutions depends on the rank $\rho$ of the linear system of $N+l-2 \kappa^{-}$real equations (5.9), (5.10), and (5.16) with respect to the $2 \kappa^{+}+1$ real unknowns $C_{0}, C_{\nu j}^{\prime}$, and $C_{\nu j}^{\prime \prime}$. Let $\tilde{\rho}=2 \kappa^{+}+1-\rho$. Clearly, if $\tilde{\rho} \leq 0$, then $\Phi(z) \equiv 0$. Otherwise, Problem 5.1 has $\tilde{\rho}$ nontrivial solutions defined by (5.6).

\subsection{Inhomogeneous case: Problem 2.3. Introduce the function}

$$
\Psi_{0}(z)=\frac{1}{4 \pi i} \int_{L} M(z, \tau) \frac{q(\tau) d \tau}{\chi^{+}(\tau)} .
$$

From the Sokhotski-Plemelj formulas and the quasimultiplicativity of the kernel $M(z, \tau)$, this function has the following properties:

$$
\begin{aligned}
& \Psi_{0}^{+}(t)-\Psi_{0}^{-}(t)=\frac{q(t)}{2 \chi^{+}(t)}, \\
& \Psi_{0}\left(\sigma_{\nu}(z)\right)=H_{\nu} \Psi_{0}(z)+\Psi_{0}\left(\sigma_{\nu}\left(z_{0}\right)\right) .
\end{aligned}
$$

By applying the Liouville theorem for multiplicative functions, we can derive the general solution to Problem 2.3 as

$$
\Phi(z)=\chi(z)\left[C_{0}+\Omega_{0}(z)+\overline{\Omega_{0}(T(z))}+\Psi_{0}(z)+\overline{\Psi_{0}(T(z))}\right],
$$

where $C_{0}$ is an arbitrary real constant and $\Omega_{0}(z)$ is the function (5.5). As in the homogeneous case, the function $\Phi(z)$ has to be invariant with respect to the group $\mathfrak{G}$. This is guaranteed by the following $N$ real conditions:

$$
\operatorname{Im}\left\{H_{k}^{-1 / 2}\left[C_{0}+\Omega_{0}\left(\sigma_{k}\left(z_{0}\right)\right)+\Psi_{0}\left(\sigma_{k}\left(z_{0}\right)\right)\right]\right\}=0, \quad k=1,2, \ldots, N .
$$


In the case $\kappa_{\nu}<0$, the function (5.22) has simple poles at the points $z_{\nu j}$. To remove these poles, we require that

$$
C_{0}+\Omega_{0}\left(z_{\nu j}\right)+\Psi_{0}\left(z_{\nu j}\right)+\overline{\Omega_{0}\left(T\left(z_{\nu j}\right)\right)}+\overline{\Psi_{0}\left(T\left(z_{\nu j}\right)\right)}=0 .
$$

Here $j=1,2, \ldots,-\kappa_{\nu}^{-}$, and $\nu=0,1, \ldots, N$. Notice that in the case $-1<\alpha_{\nu 1} \leq-\frac{1}{2}$, in general, the function $\Phi(z)$ has a nonintegrable singularity at the points $t_{\nu 1}$. The function $\Phi(z)$ becomes integrable in this case if we put

$$
l_{\nu} \operatorname{Re}\left\{H_{\nu}^{-1 / 2}\left[C_{0}+2 \Omega_{0}\left(t_{\nu 1}\right)+2 \Psi_{0}\left(t_{\nu 1}\right)-\Omega_{0}\left(\sigma_{\nu}\left(z_{0}\right)\right)-\Psi\left(\sigma_{\nu}\left(z_{0}\right)\right)\right]\right\}=0,
$$

where $\nu=0,1, \ldots, N$, and $l_{\nu}$ is given by (5.12).

Having now written down the linear system of $N+l-2 \kappa^{-}$real equations (5.23) to (5.25) for $2 \kappa^{+}+1$ real constants, $C_{0}, C_{\nu j}^{\prime}=\operatorname{Re} C_{\nu j}$ and $C_{\nu j}^{\prime \prime}=\operatorname{Im} C_{\nu j}\left(j=1,2, \ldots, \kappa_{\nu}^{+}\right.$, $\nu=0,1, \ldots, N)$, we can study its solvability. The difference between this system and that in the homogeneous case is that now the equations are not homogeneous.

If $\mathfrak{K}<0$, then the associated homogeneous system has only a trivial solution. In the inhomogeneous case, we can exclude all the constants $C_{0}, C_{\nu j}^{\prime}$, and $C_{\nu j}^{\prime \prime}$ from the system (5.23) to (5.25). This leaves us with a new system of $N+l-2 \kappa^{-}-2 \kappa^{+}-1\left(\kappa^{+}+\kappa^{-}=\kappa\right)$ conditions. If the function $q(t)=\frac{2 c(t)}{a(t)-i b(t)}$ in (2.13) satisfies these conditions, then the solution to Problem 2.3 exists and it is unique.

If $\mathfrak{K}>2 N-2$, then the rank of the system (5.23) to (5.25) coincides with the number of the equations. Therefore, the system is always solvable and the general solution to Problem 2.3 possesses $2 \kappa-l-N+1$ arbitrary real constants.

In the case $0 \leq \mathfrak{K} \leq 2 N-2$, the number of solutions and the number of solvability conditions depends on the rank $\rho\left(1 \leq \rho \leq N-2 \kappa^{-}+l\right)$ of the system (5.23) to (5.25). If the solvability conditions are met, then the general solution may have up to $\mathfrak{K}-N+1$ arbitrary real constants.

Thus we have proved the following result.

Theorem 5.3. If the index $\mathfrak{K}>2 N-2$, then Problem 2.3 is always solvable, the general solution possesses $\mathfrak{K}-N+1$ arbitrary real constants, and it is given by formula (5.22).

If $\mathfrak{K}<0$, then Problem 2.3 is solvable if and only if the functions $a(t), b(t)$, and $c(t)$ satisfy a system of $N-\mathfrak{K}-1$ conditions taken out of $N+l-2 \kappa^{-}$equations (5.23) to (5.25). If these conditions are satisfied, then the solution (5.22) to Problem 2.3 is unique.

If $0 \leq \mathfrak{K} \leq 2 N-2$, then the number of additional conditions from the system does not exceed $N-\mathfrak{K}-1$. If these conditions are met, then the solution to Problem 2.3 exists, and the number of arbitrary real constants does not exceed $\mathfrak{K}-N+1$.

Notice that the theory of solvability to the Hilbert problem (Problem 2.1) coincides with that of Problem 2.3. The general solution to Problem 2.1 is also given by formula (5.22), where we should put $z \in D$.

6. The general solution in terms of an automorphic canonical function. In this section we derive another form of the solution to Problem 2.3. Instead of the multiplicative canonical function $\chi(z)$ we shall use a piece-wise meromorphic $\mathfrak{G}$-automorphic 
canonical function. This function would be a particular case of the multiplicative canonical $\chi(z)$ function (3.14) with factors $H_{\sigma}=1, \sigma \in \mathfrak{G}$ if it did not have extra poles:

$$
\chi_{a}(z)=\exp \left\{\Gamma_{a}(z)+\overline{\Gamma_{a}(T(z))}\right\},
$$

where

$$
\Gamma_{a}(z)=\Gamma(z)+\sum_{\nu=1}^{N}\left(\int_{r_{\nu}}^{q_{\nu}} K(z, \tau) d \tau+\lambda_{\nu} \int_{L_{\nu}} K(z, \tau) d \tau+\mu_{\nu} \int_{T\left(t_{\nu 1}\right)}^{t_{\nu 1}} K(z, \tau) d \tau\right),
$$

$\Gamma(z)$ is given by (3.13) $, r_{\nu}, q_{\nu} \in D$, and $\lambda_{\nu}$ and $\mu_{\nu}$ are integers. The points $r_{\nu}$ are fixed arbitrarily while $q_{\nu}, \lambda_{\nu}$, and $\mu_{\nu}$ are to be determined. It is assumed that all the points $r_{\nu}$ and $q_{\nu}(\nu=1,2, \ldots, N)$ are distinct, and none of them coincides with the points $z_{\nu j}$ $\left(j=1,2, \ldots,\left|\kappa_{\nu}\right|, \nu=0,1, \ldots, N\right)$. It is clear that the function $\chi_{a}(z)$ possesses the properties (i) to (iv) in Definition 3.1 of the multiplicative function $\chi(z)$. Similarly to the function $\chi(z)$,

$$
\chi_{a}\left(\sigma_{k}(z)\right)=\hat{H}_{k}^{-1} \chi_{a}(z), \quad k=1,2, \ldots, N
$$

where the new factors $\hat{H}_{k}$ are given by

$$
\hat{H}_{k}=\exp \left\{-2 i \operatorname{Im} \Gamma_{a}\left(\sigma_{k}\left(z_{*}\right)\right)\right\} .
$$

The function $\chi_{a}(z)$ is invariant with respect to the group $\mathfrak{G}$ if and only if

$$
\operatorname{Im} \Gamma_{a}\left(\sigma_{k}\left(z_{*}\right)\right) \equiv 0 \quad(\bmod \pi), \quad k=1,2, \ldots, N,
$$

or, equivalently,

$$
\operatorname{Re}\left[\frac{1}{2 \pi i} \Gamma_{a}\left(\sigma_{k}\left(z_{*}\right)\right)\right] \equiv 0 \quad\left(\bmod \frac{1}{2}\right), \quad k=1,2, \ldots, N .
$$

Show next that the conditions (6.6) can be considered as the real part of the classical Jacobi inversion problem for the genus- $N$ Riemann surface $\mathfrak{R}$. Let $r_{0}$ be a fixed point in the domain $D$. Introduce the integrals

$$
\varphi_{k}(z)=\frac{1}{2 \pi i} \int_{r_{0}}^{z} \eta_{k}(\tau) d \tau, \quad k=1,2, \ldots, N
$$

where $\eta_{k}(\tau)=K\left(\sigma_{k}\left(z_{*}\right), \tau\right)$. These integrals form the normalized basis of abelian integrals of the first kind with $A$ - and $B$-periods defined in (3.4). By using (6.7) and (3.4), we can transform the conditions (6.6) as follows:

$$
\sum_{j=1}^{N}\left[\operatorname{Re} \varphi_{k}\left(q_{j}\right)+\mu_{j} \operatorname{Re} B_{k j}\right]+\lambda_{k} \equiv \operatorname{Re} d_{k} \quad\left(\bmod \frac{1}{2}\right), \quad k=1,2, \ldots, N,
$$

where

$$
d_{k}=-\frac{1}{2 \pi i} \Gamma\left(\sigma_{k}\left(z_{*}\right)\right)+\sum_{j=1}^{N} \varphi_{k}\left(r_{j}\right), \quad k=1,2, \ldots, N .
$$

Consider now another problem, a modulo-period-1 problem,

$$
\sum_{j=1}^{N}\left[\operatorname{Re} \varphi_{k}\left(q_{j}\right)+\mu_{j} \operatorname{Re} B_{k j}\right] \equiv \operatorname{Re} d_{k} \quad(\bmod 1), \quad k=1,2, \ldots, N .
$$


Evidently, each solution to the system (6.10) is a solution to the system (6.8). The new system (6.10) can be treated as the "real part" of the classical Jacobi inversion problem for the surface $\mathfrak{R}$ :

$$
\sum_{j=1}^{N}\left[\varphi_{k}\left(q_{j}\right)+\mu_{j} B_{k j}\right] \equiv \operatorname{Re} d_{k}+i \epsilon_{k} \quad(\bmod 1), \quad k=1,2, \ldots, N,
$$

where $\epsilon_{k}$ are arbitrary real numbers. It is known [13] that the solution to this problem, the points $q_{k}$ and the integers $\mu_{k}$, exist, and its solution can be expressed through the zeros of the associated genus- $N$ Riemann theta function [26, [3]. Note that the numbers $\epsilon_{k}$ can always be chosen such that the points $q_{k}$ coincide with none of the points $r_{k}$ $(k=1,2, \ldots, N)$ or $z_{\nu j}\left(j=1,2, \ldots,\left|\kappa_{\nu}\right|, \nu=0,1, \ldots, N\right)$.

The new canonical function $\chi_{a}(z)$, given by (6.1) and (6.2), is invariant with respect to the group $\mathfrak{G}, \chi_{a}(\sigma(z))=\chi_{a}(z), z \in \mathfrak{D} \backslash \mathfrak{L}$. Another difference between this function and the multiplicative function $\chi(z)$ is the presence of extra zeros and poles of the function $\chi_{a}(z)$. At the points $q_{k}$ and $T\left(q_{k}\right)$, the function $\chi_{a}(z)$ has simple zeros, and the points $r_{k}$ and $T\left(r_{k}\right)$ are simple poles $(k=1,2, \ldots, N)$.

We now repeat the procedure of Section 5 , adjusting it to the class of symmetric piecewise meromorphic multiplicative functions with factors $H_{k}=1$. The general solution to Problem 2.3 has the form

$$
\Phi(z)=\chi_{a}(z)\left[C_{0}+\Omega_{a}(z)+\Psi_{a}(z)+\overline{\Omega_{a}(T(z))}+\overline{\Psi_{a}(T(z))}\right],
$$

where

$$
\begin{gathered}
\Omega_{a}(z)=\sum_{\nu=0}^{N} \sum_{j=1}^{\kappa_{\nu}^{+}} C_{\nu j} K\left(z, z_{\nu j}\right)+\sum_{j=1}^{N} A_{j} K\left(z, q_{j}\right), \\
\Psi_{a}(z)=\frac{1}{4 \pi i} \int_{L} K(z, \tau) \frac{q(t) d \tau}{\chi_{a}(\tau)} .
\end{gathered}
$$

In comparison to the solution (5.22), the new solution (6.12) has $N$ extra complex arbitrary constants $A_{j}$, and in total it has $2 \kappa^{+}+2 N+1$ real constants. The conditions of solvability of the problem consist of $N+l-2 \kappa^{-}$real equations (5.23) to (5.25), where we should put $H_{k}=1$ and replace the functions $\Omega_{0}(z)$ and $\Psi_{0}(z)$ by the functions $\Omega_{a}(z)$ and $\Psi_{a}(z)$, respectively. In addition, to remove the simple poles at $r_{j}$ and $T\left(r_{j}\right)$ of the automorphic canonical function $\chi_{a}(z)$, we require

$$
C_{0}+\Omega_{a}\left(r_{j}\right)+\Psi_{a}\left(r_{j}\right)+\overline{\Omega_{a}\left(r_{j}\right)}+\overline{\Psi_{a}\left(T\left(r_{j}\right)\right)}=0, \quad j=1,2, \ldots, N .
$$

This brings us $2 N$ extra real conditions and makes the difference between the number of constants and the number of solvability conditions invariant to the analogue of the Cauchy kernel chosen.

7. Piecewise constant coefficients $a(t)$ and $b(t)$ : The solution for the first class group $\mathfrak{G}$. In this section we consider a particular case when the coefficients $a_{\nu}(t)$ and $b_{\nu}(t), t \in L_{\nu}(\nu=0,1, \ldots, N)$, are piecewise constant. If, in addition, the group $\mathfrak{G}$ 
is a first-class group, the formula for the multiplicative canonical function $\chi(z)$ can be simplified. Let

$$
\begin{aligned}
& a_{\nu}(t)=a_{\nu j}=\mathrm{const}, \quad b_{\nu}(t)=b_{\nu j}=\mathrm{const}, \\
& t \in t_{\nu j} t_{\nu j+1}, \quad j=1,2, \ldots, m_{\nu}, \quad \nu=0,1, \ldots, N, \quad t_{\nu m_{\nu}+1}=t_{\nu 1} .
\end{aligned}
$$

In this case $p(\tau)$ is also a piecewise constant function,

$$
p(\tau)=p_{\nu j}=-\frac{a_{\nu j}+i b_{\nu j}}{a_{\nu j}-i b_{\nu j}}, \quad \tau \in t_{\nu j} t_{\nu j+1}, \quad j=1,2, \ldots, m_{\nu}, \quad \nu=0,1, \ldots, N .
$$

According to the inequalities (3.9), (3.11), and (3.12), the values of the piecewise function $\arg p(\tau)=\arg p_{\nu j}$ and the integers $\kappa_{\nu}$ are defined by

$$
\begin{gathered}
-\pi<\arg p_{\nu 1} \leq \pi, \\
-2 \pi<\arg p_{\nu j-1}-\arg p_{\nu j} \leq 0, \quad j=2, \ldots, m_{\nu}, \quad \nu=0,1, \ldots, N, \\
-4 \pi<\arg p_{\nu m_{\nu}}-\arg p_{\nu 1}-4 \pi \kappa_{\nu} \leq 0, \quad \nu=0,1, \ldots, N .
\end{gathered}
$$

Assuming that $\mathfrak{G}$ is a first-class group, evaluate the integrals in (3.13). In this case, the kernel $K(z, \tau)$ is a uniformly and absolutely convergent series (3.7), and formula (3.13) reads

$$
\Gamma(z)=\frac{1}{4 \pi} \sum_{\nu=0}^{N} \sum_{j=1}^{m_{\nu}} \arg p_{\nu j} \int_{t_{\nu j}}^{t_{\nu j+1}} \sum_{\sigma \in \mathfrak{G}} \frac{\sigma^{\prime}(\tau)}{\sigma(\tau)-z} d \tau+\sum_{\nu=0}^{N} \operatorname{sgn} \kappa_{\nu} \sum_{j=1}^{\left|\kappa_{\nu}\right|} \int_{t_{\nu 1}}^{z_{\nu j}} \frac{\sigma^{\prime}(\tau)}{\sigma(\tau)-z} d \tau .
$$

Evaluating the integrals and using formulas (3.16), we can write

$$
\Gamma(z)=\ln \prod_{\sigma \in \mathfrak{G}} \prod_{\nu=0}^{N} \prod_{j=1}^{m_{\nu}}\left(\sigma\left(t_{\nu j}\right)-z\right)^{\alpha_{\nu j}} \prod_{j=1}^{\left|\kappa_{\nu}\right|}\left(\sigma\left(z_{\nu j}\right)-z\right)^{\operatorname{sgn} \kappa_{\nu}} .
$$

For the canonical function $\chi(z)$, we also need $\Gamma(T(z))$. Since $t_{\nu j} \in L_{\nu}$, we have $t_{\nu j}=$ $T\left(t_{\nu j}\right)$. Making the substitution $T \sigma T_{\nu}=\omega \in \mathfrak{G}$ and $T \sigma T=\omega_{*} \in \mathfrak{G}$, we can establish the following relations:

$$
\begin{aligned}
& \overline{\sigma\left(t_{\nu j}\right)-T(z)}=\frac{\rho_{0}^{2}\left(z-\omega\left(t_{\nu j}\right)\right)}{\left(\omega\left(t_{\nu j}\right)-\delta_{0}\right)\left(z-\delta_{0}\right)}, \\
& \overline{\sigma\left(z_{\nu j}\right)-T(z)}=\frac{\rho_{0}^{2}\left(z-\omega_{*}\left(z_{\nu j}^{*}\right)\right)}{\left(\omega_{*}\left(z_{\nu j}^{*}\right)-\delta_{0}\right)\left(z-\delta_{0}\right)} .
\end{aligned}
$$

Here $z_{\nu j}^{*}=T\left(z_{\nu j}\right)$. Combining the two equalities in (7.6) with the expression for $\overline{\Gamma(T(z))}$ obtained from (7.5), we derive the canonical function (3.14)

$$
\chi(z)=\left(\frac{\rho_{0}^{2}}{z-\delta_{0}}\right)^{\gamma} \Pi(z)
$$


where

$$
\begin{aligned}
\gamma & =\sum_{\nu=0}^{N}\left(\sum_{j=1}^{m_{\nu}} \alpha_{\nu j}+\kappa_{\nu}\right), \\
\Pi(z) & =\prod_{\sigma \in \mathfrak{G}} \prod_{\nu=0}^{N}\left[\prod_{j=1}^{m_{\nu}}\left(\frac{\left(\sigma\left(t_{\nu j}\right)-z\right)^{2}}{\delta_{0}-\sigma\left(t_{\nu j}\right)}\right)^{\alpha_{\nu j}} \prod_{j=1}^{\left|\kappa_{\nu}\right|}\left(\frac{\left(z-\sigma\left(z_{\nu j}\right)\right)\left(z-\sigma\left(z_{\nu j}^{*}\right)\right)}{\delta_{0}-\sigma\left(z_{\nu j}^{*}\right)}\right)^{\operatorname{sgn} \kappa_{\nu}}\right] .
\end{aligned}
$$

This formula can be further simplified. Indeed, from the definition of the numbers $\alpha_{\nu j}$ (3.16) and from the first formula in (7.8) we derive $\gamma=0$, and therefore $\chi(z)=\Pi(z)$.

ExAmPLE 7.1. Consider a particular case of Problem 2.1 when all $m_{\nu}$ are even: $m_{\nu}=$ $2 n_{\nu}(\nu=0,1, \ldots, N)$, and

$$
\begin{array}{ll}
\operatorname{Re} \phi(t)=c(t), & t \in t_{\nu j} t_{\nu j+1}, \quad j=1,3, \ldots, 2 n_{\nu}-1, \\
\operatorname{Im} \phi(t)=c(t), & t \in t_{\nu j} t_{\nu j+1}, \quad j=2,4, \ldots, 2 n_{\nu}
\end{array}
$$

and $c(t)$ is continuous on $t_{\nu j} t_{\nu j+1}, j=1,2, \ldots, 2 n_{\nu}$. In this case,

$$
p_{\nu j}=\left\{\begin{array}{cc}
-1, & j=1,3, \ldots, 2 n_{\nu}-1, \\
1, & j=2,4, \ldots, 2 n_{\nu} .
\end{array}\right.
$$

From the definition of $\arg p_{\nu j}(7.3)$ and the numbers $\kappa_{\nu}$,

$$
\arg p_{\nu j}=\pi j, \quad j=1,2, \ldots, 2 n_{\nu}, \quad \kappa_{\nu}=\left[\frac{n_{\nu}+1}{2}\right],
$$

where $[a]$ is the integer part of a number $a$. This implies

$$
\alpha_{\nu 1}=\left\{\begin{array}{l}
-3 / 4, n_{\nu}=2 s_{\nu}-1, \\
-1 / 4, \quad n_{\nu}=2 s_{\nu},
\end{array} \quad \alpha_{\nu j}=-\frac{1}{4}, \quad j=2,3, \ldots, 2 n_{\nu}, \quad \nu=0,1, \ldots, N .\right.
$$

We now observe that $\kappa_{\nu}^{-}=0, \kappa_{\nu}^{+}=\kappa_{\nu}=\left[\left(n_{\nu}+1\right) / 2\right]$.

Simple computations show that in both cases, $\alpha_{\nu 1}=-\frac{3}{4}$ and $\alpha_{\nu 1}=-\frac{1}{4}$; the index of the problem is $\mathfrak{K}=\sum_{\nu=0}^{N} n_{\nu}$. The general solution (5.22) possesses $\mathfrak{K}+l+1$ arbitrary constants $C_{0}$ and $C_{\nu j}, \nu=0,1, \ldots, N, j=1,2, \ldots, \kappa_{\nu}$. The solution has to satisfy $N+l$ conditions of solvability (5.23) and (5.25). The difference between the number of the arbitrary constants and the number of the conditions is $\mathfrak{K}+1-N$.

\section{Circular $(N+1)$-connected Hall plate with electrodes and dielectrics.}

8.1. Statement of the problem. Consider a semiconductor $D$, an infinite $N+1$-connected circular plate with finite contacts on the circles $L_{\nu}$ which form the boundary of the structure (Fig. 2). We assume that on the circles $L_{\nu}\left(\nu=0,1, \ldots, N_{0}\right)$, the number of the electrodes is even, $n_{\nu}=2 s_{\nu}$, and on the other circles $L_{\nu}\left(\nu=N_{0}+1, N_{0}+2, \ldots, N\right)$, the number of electrodes is odd, $n_{\nu}=2 s_{\nu}-1$. Here $N_{0} \in[-1, N]$. If $N_{0}=-1$, then all the circles have an odd number of electrodes. If $N_{0}=N$, then all the circles have an even number of electrodes. Let the $j$-th electrode on the circle $L_{\nu}$ be denoted as $e_{\nu j}=t_{\nu 2 j-1} t_{\nu 2 j}, j=1,2, \ldots, n_{\nu}$. It is assumed that the rest of the boundary of each circle is insulated. 


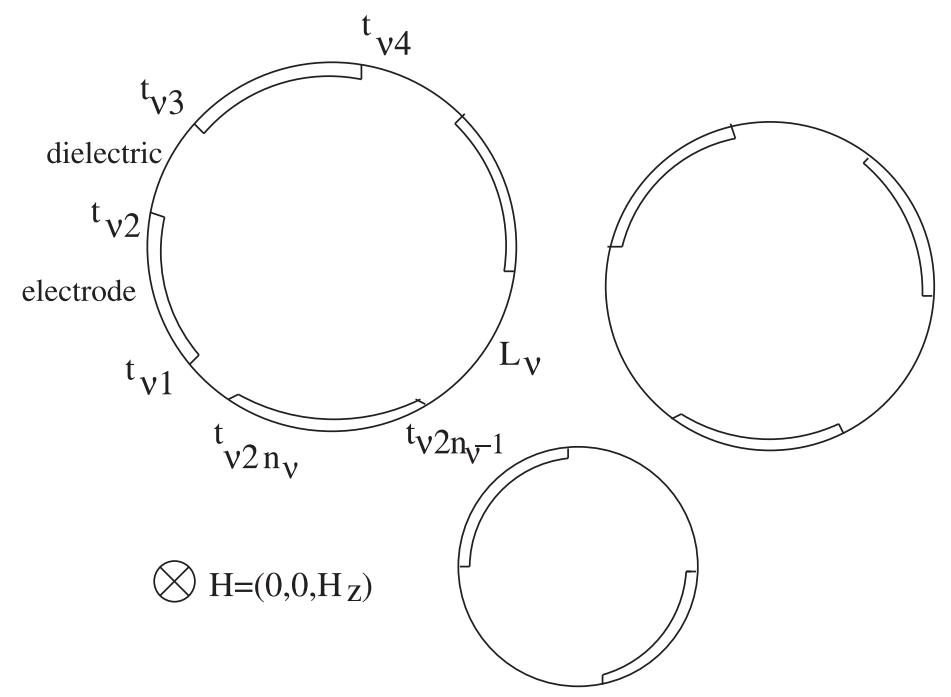

FIG. 2. An infinite multiply connected Hall plate.

Let the magnetic field be orthogonal to the plate, and its intensity $\mathbf{H}$ be prescribed, $\mathbf{H}=\left(0,0, H_{z}\right), H_{z}=$ const. The system is activated by applied electric field flowing through the electrodes

$$
J_{\nu j}=h_{0} \int_{e_{\nu j}} J_{n} d \tau, \quad j=1,2, \ldots, n_{\nu}, \quad \nu=0,1, \ldots, N .
$$

Here $h_{0}$ is the thickness of the plate, $J_{n}$ is the normal component of the current density $\mathbf{J}=\left(J_{x}, J_{y}, 0\right)$, and $J_{\nu j}$ are the total currents flowing through the electrodes. Assume also that at infinity there is no source of an external current. Then the electric field intensity $\mathbf{E}=\left(E_{x}, E_{y}, 0\right)$ vanishes at infinity,

$$
\mathbf{E}=\frac{\mathbf{A}_{0}}{z}+O\left(z^{-2}\right), \quad z \rightarrow \infty,
$$

where $\mathbf{A}=\left(A_{x}, A_{y}, 0\right)$ is a constant nonzero vector, and the currents $J_{\nu j}$ have to be prescribed such that

$$
\sum_{\nu=0}^{N} \sum_{j=1}^{n_{\nu}} J_{\nu j}=0
$$

Because of the applied electric and magnetic fields, the semiconductor develops a component of electric field orthogonal to both the electric and magnetic fields. This phenomenon, known as the Hall effect, is described by the generalized Ohm's law

$$
\mathbf{E}=\alpha \mathbf{J}-R_{H} \mathbf{J} \wedge \mathbf{H},
$$

where $\alpha$ is the resistivity in the absence of the magnetic field, and $R_{H}$ is the Hall coefficient. The Maxwell equations written for a source-free 2-d medium in the steady-state case imply the harmonicity of the current in the domain $D$. On the electrodes, the 
tangential component $E_{\tau}$ of the electric field intensity vanishes, whilst on the dielectrics (insulated walls), the normal component $J_{n}$ of the current intensity vanishes:

$$
\begin{aligned}
& -E_{x} \sin \theta+E_{y} \cos \theta=0, \quad t \in e_{\nu}, \\
& J_{x} \cos \theta+J_{y} \sin \theta=0, \quad t \in d_{\nu},
\end{aligned}
$$

where $\theta$ is the polar angle in the parametrization of the circle $L_{\nu}, t-\delta_{\nu}=\rho_{\nu} e^{i \theta}, e_{\nu}$ and $d_{\nu}$ are the unions of the electrodes and the dielectrics on the circle $L_{\nu}$, respectively,

$$
e_{\nu}=\bigcup_{j=1,3, \ldots}^{2 n_{\nu}-1} t_{\nu j} t_{\nu j+1}, \quad d_{\nu}=\bigcup_{j=2,4, . .}^{2 n_{\nu}} t_{\nu j} t_{\nu j+1},
$$

and $t_{\nu 2 n_{\nu}+1}=t_{\nu 1}$. By employing Ohm's law (8.4), we rewrite the first boundary condition in (8.5) in the form

$$
\left(\alpha \sin \theta+R_{H} \cos \theta\right) J_{x}-\left(\alpha \cos \theta-R_{H} \sin \theta\right) J_{y}=0 .
$$

Introduce next a new function, $\phi(z)=J_{x}-i J_{y}$, analytic in the domain $D$ and satisfying the Hilbert boundary condition

$$
a(t) u(t)+b(t) v(t)=0,
$$

where

$$
\begin{aligned}
& u(t)=J_{x}, \quad v(t)=-J_{y}, \\
& a(t)=\left\{\begin{array}{cc}
(\alpha+i \beta)\left(t-\delta_{\nu}\right)^{2}-(\alpha-i \beta) \rho_{\nu}^{2}, & t \in e_{\nu}, \\
\left(t-\delta_{\nu}\right) \rho_{\nu}^{-1}+\rho_{\nu}\left(t-\delta_{\nu}\right)^{-1}, & t \in d_{\nu},
\end{array}\right. \\
& b(t)=\left\{\begin{array}{cc}
i\left[(\alpha+i \beta)\left(t-\delta_{\nu}\right)^{2}+(\alpha-i \beta) \rho_{\nu}^{2}\right], & t \in e_{\nu}, \\
i\left[\left(t-\delta_{\nu}\right) \rho_{\nu}^{-1}-\rho_{\nu}\left(t-\delta_{\nu}\right)^{-1}\right], & t \in d_{\nu},
\end{array}\right.
\end{aligned}
$$

$\beta=H_{z} R_{H}$. Clearly, (8.8) is a particular case $(c(t)=0)$ of the boundary condition (2.2). The function $\phi(z)$ is sought in the class of functions which are holomorphic in $D$ and $H$ continuous in $D \cup L$ except for the points of the set $\Theta=\cup_{\nu=0}^{N} \Theta_{\nu}, \Theta_{\nu}=\left\{t_{\nu 1}, \ldots, t_{\nu 2 n_{\nu}}\right\}$, where it may have integrable singularities.

8.2. Solution to the Hilbert problem. As was shown in Section 2 for the general case, the Hilbert problem (8.8) is equivalent to the homogeneous Riemann-Hilbert problem

$$
\Phi^{+}(t)=p(t) \Phi^{-}(t), \quad t \in L \backslash \Theta
$$

for the function $\Phi(z)$ defined in (2.6). The coefficient in (8.10) is $p(t)=p_{1}(t) p_{2}(t)$, where $p_{1}(t)$ is a continuous function and $p_{2}(t)$ is a piecewise constant function given by

$$
\begin{aligned}
p_{1}(t) & =-\frac{\rho_{\nu}^{2}}{\left(t-\delta_{\nu}\right)^{2}}, \quad t \in L_{\nu}, \\
p_{2}(t) & =p_{\nu j}, \quad t \in t_{\nu j} t_{\nu j+1}, \quad j=1,2, \ldots, 2 n_{\nu}, \\
p_{\nu j} & =\left\{\begin{array}{cc}
-(\alpha-i \beta)(\alpha+i \beta)^{-1}, & j=1,3, \ldots, 2 n_{\nu}-1, \\
1, & j=2,4, \ldots, 2 n_{\nu} .
\end{array}\right.
\end{aligned}
$$

In addition, because of the condition (8.2), it is required that the solution has the following asymptotics at infinity:

$$
\Phi(z)=\frac{K}{z}+O\left(z^{-2}\right), \quad z \rightarrow \infty,
$$


where $K$ is a nonzero constant.

We split the canonical function of the problem as follows:

$$
\chi(z)=\chi_{1}(z) \chi_{2}(z)
$$

where the first function, $\chi_{1}(z)$, factorizes the continuous function $p_{1}(t)$, and the second one factorizes the piecewise constant function $p_{2}(t)$. In order to find the function $\chi_{1}(z)$, choose the starting point $t_{\nu 1} \in L_{\nu}$. Then the function $\chi_{1}(z)$ is determined by

$$
\chi_{1}(z)=\chi_{*}(z) \exp \left\{\Gamma_{1}(z)+\overline{\Gamma_{1}(T(z))}\right\},
$$

where $\chi_{*}(z)$ is a piecewise automorphic function in the domain $\mathfrak{D} \backslash \mathfrak{L}$ such that

$$
\chi_{*}(z)=\left\{\begin{array}{c}
i, \quad z \in \sigma(D), \\
-i, \quad z \in \sigma(T(D)), \quad \sigma \in \mathfrak{G} .
\end{array}\right.
$$

This function satisfies the boundary condition $\chi_{*}(t)=-\chi_{*}(t), t \in L_{\nu}(\nu=0,1, \ldots, N)$, and the symmetry and automorphicity conditions (2.9) and (2.7). The function $\Gamma_{1}(z)$ is determined by the singular integrals

$$
\Gamma_{1}(z)=\sum_{\nu=0}^{N} \frac{1}{2 \pi i} \int_{L_{\nu}} \ln \frac{\rho_{\nu}}{\tau-\delta_{\nu}} K(z, \tau) d \tau
$$

where a branch of the logarithmic function $\ln \left[\rho_{\nu}\left(z-\delta_{\nu}\right)^{-1}\right]$ is fixed in the $z$-plane cut along a line joining the branch points $z=\delta_{\nu}$ and $z=\infty$ and passing through the point $t_{\nu 1}$. The function $\chi_{1}(z)$ is a piecewise $\mathfrak{G}$-multiplicative function,

$$
\chi_{1}\left(\sigma_{j}(z)\right)=\left[H_{j}^{(1)}\right]^{-1} \chi_{1}(z), \quad j=1,2, \ldots, N,
$$

where

$$
H_{j}^{(1)}=\exp \left\{-2 i \operatorname{Im} h_{j}^{(1)}\right\}, \quad h_{j}^{(1)}=\sum_{\nu=0}^{N} \frac{1}{2 \pi i} \int_{L_{\nu}} \ln \frac{\rho_{\nu}}{\tau-\delta_{\nu}} \eta_{j}(\tau) d \tau .
$$

For the first class groups, the integrals in the expression for the function $\chi_{1}(z)$ can be evaluated. By choosing $z_{*}=\infty$, we obtain [5]

$$
\chi_{1}(z)=A_{1} \frac{\chi_{*}(z) q(z)}{z-\delta_{0}}, \quad z \in D \cup T(D),
$$

where

$$
\begin{gathered}
q(z)=\left(z-t_{01}\right) \prod_{\nu=1}^{N} \frac{z-t_{\nu 1}}{z-\delta_{\nu}} \prod_{j=0}^{N}\left(\prod_{\sigma \in \mathfrak{G}_{j}^{\prime}} \frac{z-\sigma\left(t_{j 1}\right)}{z-\sigma(\infty)} \prod_{\sigma \in \mathfrak{G}_{j}^{\prime \prime}} \frac{z-\sigma\left(t_{j 1}\right)}{z-\sigma\left(\delta_{j}\right)}\right), \\
A_{1}=\frac{\rho_{0}}{\sqrt{q\left(\delta_{0}\right)}},
\end{gathered}
$$

where $\mathfrak{G}_{j}^{\prime}$ is the set of all transformations $T_{m_{2 \mu}} T_{m_{2 \mu-1}} \ldots T_{m_{2}} T_{j}, m_{2} \neq j, m_{3} \neq m_{2}, \ldots$, $m_{2 \mu} \neq m_{2 \mu-1}, \mu=1,2, \ldots$, where all the indices $m_{\mu}$ vary from 0 to $n: m_{\mu}=0,1, \ldots, n$. The set $\mathfrak{G}_{j}^{\prime \prime}=\mathfrak{G} \backslash \mathfrak{G}_{j}^{\prime} \backslash \sigma_{0}$ includes all the other transformations $T_{m_{2 \mu}} T_{m_{2 \mu-1}} \ldots T_{m_{2}} T_{m_{1}}$ $\left(m_{1} \neq j\right)$ of the group $\mathfrak{G}$ except for the identical transformation $\sigma_{0}$.

Notice that the constant $A_{1}$ in the representation (8.19) of the canonical function $\chi_{1}(z)$ cannot be removed. It is needed to satisfy the symmetry condition $\chi_{1}(z)=\overline{\chi_{1}(T(z))}$. 
The branch in (8.20) is chosen arbitrarily. Its choice affects the sign of the constant and does not break the symmetry of the canonical function. We shall also need a series representation of the coefficients $h_{\nu}^{(1)}$. For the first-class group $\mathfrak{G}$, formula (8.18) yields [5]

$$
\begin{aligned}
2 h_{\nu}^{(1)}= & \ln \frac{T_{\nu}\left(\delta_{0}\right)-\delta_{\nu}}{\rho_{\nu}}+\sum_{j=0}^{N}\left[\ln \frac{T_{\nu}\left(\delta_{0}\right)-t_{j 1}}{T_{\nu}\left(\delta_{0}\right)-\delta_{j}}\right. \\
& \left.+\sum_{\sigma \in \mathfrak{G}_{j}^{\prime}} \ln \frac{T_{\nu}\left(\delta_{0}\right)-\sigma\left(t_{j 1}\right)}{T_{\nu}\left(\delta_{0}\right)-\sigma(\infty)}+\sum_{\sigma \in \mathfrak{G}_{j}^{\prime \prime}} \ln \frac{T_{\nu}\left(\delta_{0}\right)-\sigma\left(t_{j 1}\right)}{T_{\nu}\left(\delta_{0}\right)-\sigma\left(\delta_{j}\right)}\right] .
\end{aligned}
$$

In Section 7 we have determined the canonical function (7.7) and showed that $\gamma=0$. To use this formula for the function $\chi_{2}(z)$, we specify the parameters. Notice that the numbers $m_{\nu}$ are always even, $m_{\nu}=2 n_{\nu}$, and the parameter $\beta$ can be any finite real number. Introduce the parameter

$$
\delta=2 \tan ^{-1} \frac{\alpha}{|\beta|} \in(0, \pi) .
$$

First, let $\beta>0$. According to the inequalities (7.3), we choose $\arg p_{\nu j}$ as follows:

$$
\begin{aligned}
& \arg p_{\nu j}=\delta+(j-1) \pi, \quad j=1,3, \ldots, 2 n_{\nu}-1, \\
& \arg p_{\nu j}=j \pi, \quad j=2,4, \ldots, 2 n_{\nu} .
\end{aligned}
$$

It remains now to write down the integers $\kappa_{\nu}$ and the parameters $\alpha_{\nu j}$. Since the function $\chi_{1}(z)$ has simple zeros at the points $t_{\nu 1}$ it will be convenient to choose the integers $\kappa_{\nu}$ such that the following inequalities hold:

$$
-4 \pi<\arg p_{\nu 2 n_{\nu}}-\arg p_{\nu 1}-4 \pi \kappa_{\nu}+2 \pi \leq 0,
$$

from which we obtain

$$
\kappa_{\nu}=\left[\frac{n_{\nu}}{2}\right]+1=\left\{\begin{array}{c}
s_{\nu}+1, \quad n_{\nu}=2 s_{\nu} \\
s_{\nu}, \quad n_{\nu}=2 s_{\nu}-1
\end{array}\right.
$$

We also need to determine the constants $\alpha_{\nu j}$,

$$
\begin{aligned}
& \alpha_{\nu 1}=\frac{1}{4 \pi}\left(\arg p_{\nu 2 n_{\nu}}-\arg p_{\nu 1}+2 \pi\right)-\kappa_{\nu}=\left\{\begin{array}{cc}
-\delta_{*}-\frac{1}{2}, & n_{\nu}=2 s_{\nu}, \\
-\delta_{*}, & n_{\nu}=2 s_{\nu}-1,
\end{array}\right. \\
& \alpha_{\nu j}=\frac{1}{4 \pi}\left(\arg p_{\nu j-1}-\arg p_{\nu j}\right)=\left\{\begin{array}{cc}
-\delta_{*}, & j=3,5, \ldots, 2 n_{\nu}-1, \\
\delta_{*}-\frac{1}{2}, & j=2,4, \ldots, 2 n_{\nu},
\end{array}\right.
\end{aligned}
$$

where

$$
\delta_{*}=\frac{\delta}{4 \pi}, \quad \nu=0,1, \ldots, N
$$


Next, consider the case $\beta<0$. The parameters of interest are defined as follows:

$$
\begin{gathered}
\arg p_{\nu j}=-\delta+(j-1) \pi, \quad j=1,3, \ldots, 2 n_{\nu}-1, \\
\arg p_{\nu j}=(j-2) \pi, \quad j=2,4, \ldots, 2 n_{\nu}, \\
\alpha_{\nu 1}=\left\{\begin{array}{cc}
\delta_{*}-1, & n_{\nu}=2 s_{\nu}-1, \\
\delta_{*}-\frac{1}{2}, \quad n_{\nu}=2 s_{\nu},
\end{array}\right. \\
\alpha_{\nu j}=\left\{\begin{array}{c}
\delta_{*}-\frac{1}{2}, \quad j=3,5, \ldots, 2 n_{\nu}-1, \quad \nu=0,1, \ldots, N, \\
-\delta_{*}, \quad j=2,4, \ldots, 2 n_{\nu},
\end{array}\right.
\end{gathered}
$$

and the integers $\kappa_{\nu}=\left[n_{\nu} / 2\right]+1$ are the same as in the case $\beta>0$. Now, with the parameters $\alpha_{\nu j}$ and $\kappa_{\nu}$ being defined, we can write down the function $\chi_{2}(\zeta)$ :

$$
\chi_{2}(z)=\prod_{\sigma \in \mathfrak{G}} \prod_{\nu=0}^{N}\left[\prod_{j=1}^{2 n_{\nu}}\left(\frac{\left(\sigma\left(t_{\nu j}\right)-z\right)^{2}}{\delta_{0}-\sigma\left(t_{\nu j}\right)}\right)^{\alpha_{\nu j}} \prod_{j=1}^{\kappa_{\nu}}\left(\frac{\left(z-\sigma\left(z_{\nu j}\right)\right)\left(z-\sigma\left(z_{\nu j}^{*}\right)\right)}{\delta_{0}-\sigma\left(z_{\nu j}^{*}\right)}\right)\right] .
$$

The function $\chi_{2}(z)$ is the multiplicative canonical function with the factors $H_{j}^{(2)}$ obtained by replacing the factors $H_{j}$ derived in Section 3 by $H_{j}^{(2)}=\exp \left(-2 i \operatorname{Im} h_{\nu}^{(2)}\right), h_{\nu}^{(2)}=$ $\Gamma_{2}\left(\sigma_{\nu}\left(z_{*}\right)\right)$. The function $\Gamma_{2}(z)$ coincides with the function $\Gamma(z)$ in (3.13) if $p(\tau)$ is replaced by $p_{2}(\tau)$.

Having found the functions $\chi_{1}(z)$ and $\chi_{2}(z)$, we write down the general solution of the Riemann-Hilbert problem:

$$
\Phi(z)=\chi_{1}(z) \chi_{2}(z)\left[C_{0}+\Omega_{0}(z)+\overline{\Omega_{0}(T(z))}\right],
$$

where

$$
\Omega_{0}(z)=\sum_{\nu=0}^{N} \sum_{j=1}^{\kappa_{\nu}} C_{\nu j} M\left(z, z_{\nu j}\right)
$$

$M(z, \tau)$ is the quasimultiplicative kernel with the factors $H_{j}=H_{j}^{(1)} H_{j}^{(2)}, C_{0}$ is a real constant, and $C_{\nu j}=C_{\nu j}^{\prime}+i C_{\nu j}^{\prime \prime}$ are complex constants.

8.3. Definition of the constants. In total, the general solution (8.30) possesses $2 \kappa+1$ real constants to be determined. It will be convenient to have another representation of the number of the constants. Since

$$
\kappa=\sum_{\nu=0}^{N} \kappa_{\nu}, \quad \kappa_{\nu}=\left[\frac{n_{\nu}}{2}\right]+1,
$$

$n_{\nu}=2 s_{\nu}$ if $\nu=0,1, \ldots, N_{0}$, and $n_{\nu}=2 s_{\nu}-1$ if $\nu=N_{0}+1, N_{0}+2, \ldots, N$, we obtain

$$
2 \kappa+1=2 s+2 N_{0}+3, \quad s=\sum_{\nu=0}^{N} s_{\nu}
$$

Next, show that for the definition of these constants, we have the same number of linear equations. The first $N$ equations come from the conditions which guarantee that the solution $\Phi(z)$ is invariant with respect to the group $\mathfrak{G}$ :

$$
\operatorname{Im}\left\{H_{k}^{-1 / 2}\left[C_{0}+\Omega_{0}\left(T_{k}\left(\delta_{0}\right)\right)\right]\right\}=0, \quad k=1,2, \ldots, N .
$$


The point $z_{0}$ in the representation of the quasimultiplicative kernel $M(z, \tau)$ in 4.13 can be fixed arbitrarily. It is convenient to choose $z_{0}=\infty$. For this choice, $\sigma_{k}(\infty)=T_{k}\left(\delta_{0}\right)$, and the additional conditions 5.23 reduce to the form 8.34 .

The function $\Phi(z)$ has to have a simple zero at infinity. This is guaranteed by the complex condition $C_{0}+\overline{\Omega_{0}\left(\delta_{0}\right)}=0$, which is equivalent to the following two real equations:

$$
\begin{gathered}
C_{0}=-\operatorname{Re}\left\{\Omega_{0}\left(\delta_{0}\right)\right\}, \\
\operatorname{Im}\left\{\Omega_{0}\left(\delta_{0}\right)\right\}=0 .
\end{gathered}
$$

We have shown that if $n_{\nu}=2 s_{\nu}$, then the parameter $\alpha_{\nu 1}=-\delta_{*}-\frac{1}{2}$ when $\beta>0$ and $\alpha_{\nu 1}=\delta_{*}-1$ when $\beta<0$. This means that $2 \alpha_{\nu 1} \in(-2,-1)$ when the number of electrodes is even. Otherwise, $2 \alpha_{\nu 1} \in(-1,0)$. Thus, if $n_{\nu}=2 s_{\nu}$, then the solution (8.30) has a nonintegrable singularity at the points $t_{\nu 1}$. It becomes an integrable singularity if the conditions (5.16) hold. As in equations (8.34) and (8.35), we put $z_{0}=\infty$ and use the fact that $l_{\nu}=1$ if $\nu=0,1, \ldots, N_{0}$, and $l_{\nu}=0$ for $\nu>N_{0}$. This transforms equations (5.16) to the following $N_{0}+1$ conditions:

$$
\operatorname{Re}\left\{H_{\nu}^{-1 / 2}\left[C_{0}+2 \Omega_{0}\left(t_{\nu 1}\right)-\Omega_{0}\left(T_{\nu}\left(\delta_{0}\right)\right)\right]\right\}=0, \quad \nu=0,1, \ldots, N_{0} .
$$

In addition, we have the physical conditions (8.1), which can be written in the form

$$
\int_{e_{\nu j}} J_{n} d \tau=\frac{J_{\nu j}}{h_{0}}, \quad j=1,2, \ldots, n_{\nu}, \quad \nu=0,1, \ldots, N,
$$

where

$$
J_{n}=J_{x} \cos \theta+J_{y} \sin \theta, \quad J_{x}=\operatorname{Re} \Phi(z), \quad J_{y}=-\operatorname{Im} \Phi(z) .
$$

The number $n=n_{0}+\ldots+n_{N}$ of the conditions (8.37) can be expressed through the integers $s, N$, and $N_{0}$ as follows:

$$
n=2 s-N+N_{0} .
$$

Thus, we obtain a system which consists of $N$ equations (8.34), two conditions (8.35), $N_{0}+1$ relations (8.36) and $2 s-N+N_{0}$ equations (8.37). As we had anticipated, in total, there are $2 s+2 N_{0}+3$ real equations for the determination of $2 s+2 N_{0}+3$ real constants.

The first equation in (8.35) expresses the real constant $C_{0}$ through the constants $C_{\nu j}$. This makes it possible to simplify the solution of the problem. The final formula has $2 \kappa=2 s+2 N_{0}+2$ real constants, and it becomes

$$
J_{x}-i J_{y}=\sum_{\nu=0}^{N} \sum_{j=1}^{\kappa_{\nu}}\left[C_{\nu j}^{\prime} S_{\nu j}^{+}(z)+i C_{\nu j}^{\prime \prime} S_{\nu j}^{-}(z)\right], \quad z \in D,
$$

where the functions $S_{\nu j}^{ \pm}$are free of the constants $C_{\nu j}=C_{\nu j}^{\prime}+i C_{\nu j}^{\prime \prime}$, and

$$
S_{\nu j}^{ \pm}(z)=\chi_{1}(z) \chi_{2}(z)\left[-\frac{1}{2} M\left(\delta_{0}, z_{\nu j}\right) \mp \frac{1}{2} \overline{M\left(\delta_{0}, z_{\nu j}\right)}+M\left(z, z_{\nu j}\right) \pm \overline{M\left(T(z), z_{\nu j}\right)}\right] .
$$


Conclusions. We have developed a method for the Hilbert problem for a circular multiply connected domain and the Riemann-Hilbert problem for piecewise analytic functions invariant with respect to a symmetric Schottky group. The coefficients of both problems are piecewise Hölder continuous functions, and the discontinuities of the coefficients cause integrable singularities of the solution. The technique we have proposed requires the use of two analogues of the Cauchy kernel, a quasiautomorphic kernel and a quasimultiplicative kernel. We have proved the existence results for both kernels. The existence of the former kernel follows from the theory of abelian integrals on a compact Riemann surface. To prove the existence of the quasimultiplicative kernel, we have used the Riemann-Roch theorem for multiplicative functions. For the first-class groups (the Burnside classification), the solutions to the Hilbert and the Riemann-Hilbert problems have been derived in a series form. In addition, we have obtained the solution in terms of an automorphic analogue of the Cauchy kernel. It turns out that the use of this kernel requires the solution of the Jacobi inversion problem. We emphasize that the procedure which is based on the quasiautomorphic and quasimultiplicative kernels bypasses the Jacobi inversion problem. There is another advantage to employing the quasiautomorphic and quasimultiplicative kernels, not the automorphic kernel. For an $(N+1)$-connected circular domain, the second method leads to a solution which has $2 N$ extra real constants and therefore, in comparison with the first method, there are $2 N$ extra equations to be solved.

The method proposed has been illustrated by the solution of a model electromagnetic steady-state problem on the motion of charged electrons in a plate when the applied magnetic field is orthogonal to the plate. The plate, known as a Hall plate, has $N+1$ circular holes with electrodes and dielectrics on the walls. We have reduced the problem to a particular case of the Hilbert problem with the coefficient $p(t)=p_{1}(t) p_{2}(t)$. The first function is continuous, and its factorization has been implemented by the method [5]. The second function, $p_{2}(t)$, is a piecewise constant function. Because of this property and also because the first canonical function has a zero at the starting point, we have managed to simplify the general formula for the second canonical function. We have derived the exact formula for the current density. The formula possesses a finite number, $2 \kappa$, of unknown constants which solve a system of $2 \kappa$ linear algebraic equations, where $2 \kappa=2 s+2 N_{0}+2, N_{0}$ is the number of circles with an even number of electrodes, $s=s_{0}+s_{1}+\ldots s_{N}, s_{\nu}=\left[\left(n_{\nu}+1\right) / 2\right]$, and $n_{\nu}$ is the number of electrodes on the $\nu$ th circle. Note that the system of equations for the constants consists of $N+N_{0}+2$ "mathematical" equations (due to the method) and $2 s+N_{0}-N \geq 1$ physical equations.

Finally, we notice that the technique proposed can be extended for polygonal multiply connected domains. This can be done by implementing a two-step-procedure. First, one needs to map an $(N+1)$-connected circular domain into an $(N+1)$-connected polygonal domain [10, 9] and define the coefficients $a(t), b(t)$, and $c(t)$. The second step is to use the solution to the Hilbert problem for an $(N+1)$-connected circular domain derived in this paper. 


\section{REFERENCES}

[1] L.A. Aksent'ev, Construction of the Schwarz operator by the symmetry method, Trudy Sem. Kraev. Zadacham (Kazan) 4 (1967), 3-10. MR0291425 (45:518)

[2] I.A. Aleksandrov and A.S. Sorokin, The problem of Schwarz for multiply connected circular domains, Siberian Math. J. 13 (1973), 671-692.

[3] Y.A. Antipov and V.V. Silvestrov, Electromagnetic scattering from an anisotropic impedance halfplane at oblique incidence: The exact solution, Quart. J. Mech. Appl. Math. 59 (2006), 211-251. MR 2219885 (2007j:78009)

[4] Y.A. Antipov and V.V. Silvestrov, Method of automorphic functions in the study of flow around a stack of porous cylinders, Quart. J. Mech. Appl. Math. 60 (2007), 337-366. MR2347761 (2008m:35274)

[5] Y.A. Antipov and V.V. Silvestrov, Circular map for supercavitating flow in a multiply-connected domain, Quart. J. Mech. Appl. Math. 62 (2009), 167-200. MR2496027

[6] Y.A. Antipov and D.G. Crowdy, Riemann-Hilbert problem for automorphic functions and the Schottky-Klein function, Complex Anal. Oper. Theory 1 (2007), 317-334. MR2336026 (2008g:30032)

[7] W. Burnside, On a class of automorphic functions, Proc. London Math. Soc. 23 (1892), 49-88.

[8] L.I. Chibrikova and V.V. Silvestrov, On the question of the effectiveness of the solution of Riemann's boundary value problem for automorphic functions, Soviet Math. (Iz. VUZ) 12 (1978), 117-121. MR529717 (80j:30058)

[9] D. Crowdy, The Schwarz-Christoffel mapping to bounded multiply connected polygonal domains, Proc. R. Soc. A 461 (2005), 2653-2678. MR2165505 (2006h:30005)

[10] T.K. DeLillo, A.R. Elcrat and J.A. Pfaltzgraff, Schwarz-Christoffel mapping of multiply-connected domains, J. d'Analyse 94 (2005), 17-48. MR2124453 (2006b:30010)

[11] T.A. Driscoll and L.N. Trefethen, Schwarz-Christoffel Mapping, Cambridge University Press, Cambridge, 2002. MR1908657 (2003e:30012)

[12] Y.P. Emets, Boundary Value Problems of Electrodynamics for Anisotropically Conducting Media, Naukova Dumka, Kiev, 1987.

[13] H.M. Farkas and I. Kra, Riemann Surfaces, Springer-Verlag, New York, $1980 . \quad$ MR583745 (82c:30067)

[14] L.R. Ford, Automorphic Functions, AMS Chelsea Publishing, Providence, RI, 2004.

[15] F.D. Gakhov, Boundary Value Problems, Dover Publications, New York, 1990. MR.1106850 (92b:45005)

[16] J. Haeusler, Exakte Lösungen von Potentialproblemen beim Halleffekt durch konforme Abbildung, Solid-State Electronics 9 (1966), 417-441.

[17] J. Haeusler and H.J. Lippmann, Hallgeneratoren mit kleinem Linearisierungsfehler, Solid-State Electronics 11 (1968), 173-182.

[18] A. Hurvitz and R. Courant, Vorlesungen Über Allgemeine Funktiontheorie und Elliptische Funktionen, Springer-Verlag, Berlin, 1964. MR0173749 (30:3959)

[19] I. Kra, Automorphic Forms and Kleinian Groups, W.A. Benjamin, Inc., Reading, MA, 1972. MR0357775 (50:10242)

[20] V.V. Silvestrov, The Riemann boundary value problem for symmetric automorphic functions and its application, Theory of functions of a complex variable and boundary value problems, 93-107, Chuvash. Gos. Univ., Cheboksary, 1982.

[21] L.N. Trefethen and R.J. Williams, Conformal mapping solution of Laplace's equation on a polygon with oblique derivative boundary conditions, J. Comp. Appl. Math. 14 (1986), 227-249. MR829041 (87f:30019)

[22] I.N. Vekua, Generalized Analytic Functions, Pergamon, New York, 1962. MR.0150320(27:321)

[23] W. Versnel, Analysis of Hall-plate shaped Van der Pauw structures, Solid-State Electronics 23 (1980), 557-563.

[24] W. Versnel, Analysis of a circular Hall plate with equal finite contacts, Solid-State Electronics 24 (1981), 63-68.

[25] R.V. Wick, Solution of the field problem of the germanium gyrator, J. Appl. Physics 25 (1954), $741-756$.

[26] E.I. Zverovich, Boundary value problems in the theory of analytic functions in Hölder classes on Riemann surfaces, Russian Math. Surveys 26 (1971), 117-192. MR0409841 (53:13593) 